\title{
The Mellin transform of powers of the zeta-function
}

\author{
by
}

\section{Aleksandar Ivić (Beograd), Matti Jutila (Turku) and Yoichi MотоHashi (Tokyo)}

1. Introduction. Let as usual

$$
F(s):=\int_{0}^{\infty} f(x) x^{s-1} d x
$$

with $s=\sigma+i t$ denote the Mellin transform of $f(x)$. Mellin transforms play a fundamental rôle in Analytic Number Theory. They can be viewed, by a change of variable, as special cases of Fourier transforms, and their properties can be deduced from the general theory of Fourier transforms. For an extensive account, we refer the reader to E. C. Titchmarsh [25]. One of the basic properties of Mellin transforms is the inversion formula

$$
\frac{1}{2}\{f(x+0)+f(x-0)\}=\frac{1}{2 \pi i} \int_{(\sigma)} F(s) x^{-s} d s=\frac{1}{2 \pi i} \lim _{T \rightarrow \infty} \int_{\sigma-i T}^{\sigma+i T} F(s) x^{-s} d s .
$$

This holds, for example, if $f(x) x^{\sigma-1} \in L(0, \infty)$, and $f(x)$ is of bounded variation on every finite $x$-interval. We remark that if $G(s)$ denotes the Mellin transform of $g(x)$, then assuming $f(x)$ and $g(x)$ to be real-valued, we formally have

$$
\begin{aligned}
\frac{1}{2 \pi i} \int_{(\sigma)} F(s) \overline{G(s)} d s & =\int_{0}^{\infty} g(x)\left(\frac{1}{2 \pi i} \int_{(\sigma)} F(s) x^{\sigma-i t-1} d s\right) d x \\
& =\int_{0}^{\infty} g(x) x^{2 \sigma-1}\left(\frac{1}{2 \pi i} \int_{(\sigma)} F(s) x^{-s} d s\right) d x \\
& =\int_{0}^{\infty} f(x) g(x) x^{2 \sigma-1} d x
\end{aligned}
$$

The relation (1.1) is a form of Parseval's formula for Mellin transforms, and

2000 Mathematics Subject Classification: Primary 11M06; Secondary 11F72. 
it offers various possibilities for mean square bounds. A condition under which (1.1) holds is that $x^{\sigma} f(x)$ and $x^{\sigma} g(x)$ belong to $L^{2}((0, \infty), d x / x)$. A variant of (1.1) is (see [25, Theorem 73])

$$
\frac{1}{2 \pi i} \int_{(c)} F(w) G(s-w) d w=\int_{0}^{\infty} f(x) g(x) x^{s-1} d x,
$$

which holds if $x^{c} f(x)$ and $x^{\sigma-c} g(x)$ belong to $L^{2}((0, \infty), d x / x)$.

The main object of study in this work is the Mellin transform function

$$
\mathcal{Z}_{k}(s):=\int_{1}^{\infty}|\zeta(1 / 2+i x)|^{2 k} x^{-s} d x
$$

which is a regular function of $s$ for any given $k \geq 0$ and $\sigma \geq \sigma_{0}(k)(>1)$. However from the viewpoint of possible applications it is expedient to assume that $k \in \mathbb{N}$. Note that the lower bound of integration in (1.3) is not zero, as is customary in the definitions of Mellin transforms. The choice of unity as the lower bound of integration dispenses with convergence problems at that point, while the appearance of the factor $x^{-s}$ instead of the customary $x^{s-1}$ is technically more convenient. Also it may be compared with the discrete representation

$$
\zeta^{2 k}(s)=\sum_{n=1}^{\infty} d_{2 k}(n) n^{-s} \quad(\sigma>1)
$$

where $d_{m}(n)$ is the number of ways $n$ may be written as a product of $m$ factors; $d(n) \equiv d_{2}(n)$ is the number of divisors of $n$. Since we have (see [4, Chapter 8])

$$
\int_{0}^{T}|\zeta(1 / 2+i t)|^{2 k} d t \ll T^{(k+2) / 4} \log ^{C(k)} T \quad(2 \leq k \leq 6),
$$

it follows that the integral defining $\mathcal{Z}_{k}(s)$ is absolutely convergent for $\sigma>1$ if $0 \leq k \leq 2$ and for $\sigma>(k+2) / 4$ if $2 \leq k \leq 6$. In fact, the bound (1.5) is the sharpest one known, and it is a fundamental problem of zeta-function theory to improve it, in particular to prove for the sixth and the eighth moment the hypothetical estimates

$$
\int_{0}^{T}|\zeta(1 / 2+i t)|^{6} d t \ll T^{1+\varepsilon}, \quad \int_{0}^{T}|\zeta(1 / 2+i t)|^{8} d t \ll T^{1+\varepsilon},
$$

where $\varepsilon$ denotes arbitrarily small constants, not necessarily the same at each occurrence. We note that the first author in [7] showed that the sixth moment (the first bound in (1.6)) follows if a certain conjecture involving the sum $\sum_{n \leq x} d_{3}(n) d_{3}(n+h)$ holds, where $h$ is not fixed. In what concerns 
the eighth moment, N. V. Kuznetsov [16] had an approach based on the use of spectral theory, but his argument for a proof of

$$
\int_{0}^{T}|\zeta(1 / 2+i t)|^{8} d t \ll T \log ^{C} T \quad(C>0)
$$

had several gaps (see the review in Zbl.745.11040).

Perhaps more promising in the context of the eighth moment is the possibility to use (1.1), as mentioned by the third author [24, p. 216], where also the possibility of the use of the Parseval formula for the Hermite-Fourier expansion is discussed. This approach will lead to an exact identity for a weighted form of the eighth moment in terms of well defined objects from spectral theory.

One may use $(1.2)$ to derive a recurrent relation for $\mathcal{Z}_{k}(s)$, namely

$$
\mathcal{Z}_{k}(s)=\frac{1}{2 \pi i} \int_{(c)} \mathcal{Z}_{k-r}(w) \mathcal{Z}_{r}(1-w+s) d w \quad(r=1, \ldots, k-1),
$$

which is valid for $c \geq c_{k}>0$ and $\sigma=\operatorname{Re} s \geq \sigma_{1}(k)(>1)$. To see this, we take $f(x)=\left|\zeta\left(1 / 2+i x^{-1}\right)\right|^{2 k-2 r} x^{-1}, g(x)=\left|\zeta\left(1 / 2+i x^{-1}\right)\right|^{2 r} x^{-1}$ if $0<x<1$ and $f(x)=g(x)=0$ if $x>1$. Then $F(s)=\mathcal{Z}_{k-r}(s), G(s)=\mathcal{Z}_{r}(s)$, and (1.7) follows from (1.2) (with $s$ replaced by $s+1$ ). Note that the hypotheses under which (1.2) hold can be used, in conjunction with the known results on power moments of $|\zeta(1 / 2+i t)|$, to determine $c$ and $\sigma$ for which (1.7) will hold. For example, using (1.5) with $k=2$ and $k=4$, in this manner we shall obtain

$$
\mathcal{Z}_{3}(s)=\frac{1}{2 \pi i} \int_{(1+\varepsilon)} \mathcal{Z}_{1}(w) \mathcal{Z}_{2}(1-w+s) d w \quad(\sigma>5 / 4),
$$

and

$$
\mathcal{Z}_{4}(s)=\frac{1}{2 \pi i} \int_{(5 / 4+\varepsilon)} \mathcal{Z}_{2}(w) \mathcal{Z}_{2}(1-w+s) d w \quad(\sigma>3 / 2) .
$$

In what concerns power moments of $|\zeta(1 / 2+i t)|$ one expects, for any fixed $k \in \mathbb{N}$,

$$
\int_{0}^{T}|\zeta(1 / 2+i t)|^{2 k} d t=T P_{k^{2}}(\log T)+E_{k}(T)
$$

to hold, where it is generally assumed that $P_{k^{2}}(y)$ is a polynomial in $y$ of degree $k^{2}$ (the integral in (1.10) is $\gg_{k} T \log ^{k^{2}} T$; see e.g., [4, Chapter 9]). The function $E_{k}(T)$ is to be considered as the error term in (1.10), namely

$$
E_{k}(T)=o(T) \quad(T \rightarrow \infty) .
$$


So far (1.10) and (1.11) are known to hold only for $k=1$ and $k=2$ (see [5] and [23] for a comprehensive account). Therefore in view of the existing knowledge on higher moments of $|\zeta(1 / 2+i t)|$, embodied in (1.4), at present the really important cases of (1.3) are $k=1$ and $k=2$.

In Section 3 we shall study the function $\mathcal{Z}_{1}(s)$ and obtain its meromorphic continuation to the half-plane $\sigma>-3 / 4$. This will be achieved by the use of Atkinson's formula (or its integrated version) for the function $E_{1}(T) \equiv E(T)$ in (1.10). The function $\mathcal{Z}_{1}(s)$, which does not seem to have been studied yet in the literature, is regular for $\sigma>-3 / 4$, except for a pole at $s=1$ of order two. In this region it is also of polynomial growth in $|s|$, which will be convenient to have in any applications. In general, namely, if $f(x)$ is a smooth function of fast decay whose Mellin transform is $F(s)$, then by the Mellin inversion formula

$$
\begin{aligned}
\int_{1}^{\infty} f(x / T) \mid \zeta(1 / 2+ & i x)\left.\right|^{2 k} d x \\
& =\int_{1}^{\infty} \frac{1}{2 \pi i} \int_{(c)} F(s)\left(\frac{T}{x}\right)^{s} d s|\zeta(1 / 2+i x)|^{2 k} d x \\
& =\frac{1}{2 \pi i} \int_{(c)} F(s) T^{s} \mathcal{Z}_{k}(s) d s
\end{aligned}
$$

for suitable $c(>1)$. If sufficient information on $\mathcal{Z}_{k}(s)$ is available, then shifting the line of integration in (1.12), using the residue theorem etc. we can expect to acquire interesting information on the first integral in (1.12).

The use of (1.12) is especially interesting in the case $k=2$. The function $\mathcal{Z}_{2}(s)$ was introduced and studied by the third author [22], [24, Chapter 5]. He has shown that $\mathcal{Z}_{2}(s)$ has meromorphic continuation over $\mathbb{C}$. In the halfplane $\operatorname{Re} s>0$ it has the following singularities: the pole $s=1$ of order five, simple poles at $s=1 / 2 \pm i \kappa_{j}\left(\kappa_{j}=\sqrt{\lambda_{j}-1 / 4}\right)$ and poles at $s=\varrho / 2$, where $\varrho$ denotes complex zeros of $\zeta(s)$. The residue of $\mathcal{Z}_{2}(s)$ at $s=1 / 2+i \kappa_{h}$ equals

$$
\begin{aligned}
R\left(\kappa_{h}\right):=\sqrt{\frac{\pi}{2}}\left(2^{-i \kappa_{h}} \frac{\Gamma\left(1 / 4-i \kappa_{h} / 2\right)}{\Gamma\left(1 / 4+i \kappa_{h} / 2\right)}\right)^{3} & \\
\times & \times\left(2 i \kappa_{h}\right) \cosh \left(\pi \kappa_{h}\right) \sum_{\kappa_{j}=\kappa_{h}} \alpha_{j} H_{j}^{3}(1 / 2),
\end{aligned}
$$

and the residue at $s=1 / 2-i \kappa_{h}$ equals $\overline{R\left(\kappa_{h}\right)}$. Here as usual $\left\{\lambda_{j}=\kappa_{j}^{2}+\right.$ $1 / 4\} \cup\{0\}$ is the discrete spectrum of the non-Euclidean Laplacian acting on $\mathrm{SL}(2, \mathbb{Z})$-automorphic forms and $\alpha_{j}=\left|\varrho_{j}(1)\right|^{2}\left(\cosh \pi \kappa_{j}\right)^{-1}$, where $\varrho_{j}(1)$ is the first Fourier coefficient of the Maass wave form corresponding to the eigenvalue $\lambda_{j}$ to which the Hecke $L$-function $H_{j}(s)$ is attached (see [24, 
Chapters 1-3] for a comprehensive account of spectral theory and the Hecke $L$-functions). This shows that the analytic behaviours of $\mathcal{Z}_{1}(s)$ and $\mathcal{Z}_{2}(s)$ are quite different. One can use (1.8) and (1.9) to investigate the analytic behaviour of the more complicated functions $\mathcal{Z}_{3}(s), \mathcal{Z}_{4}(s)$, and (1.7) for general $\mathcal{Z}_{k}(s)$.

The function $\mathcal{Z}_{2}(s)$ was already used to furnish several strong results on $E_{2}(T)$ (see (1.10)), the error term in the asymptotic formula for the fourth moment of $|\zeta(1 / 2+i t)|$. The third author ([22] and [24, Chapter 5]) used it to show that $E_{2}(T)=\Omega_{ \pm}\left(T^{1 / 2}\right)$, which sharpens the earlier result of [9] that $E_{2}(T)=\Omega\left(T^{1 / 2}\right)$ (see also [5] and [11]). The first and third authors have shown (see [5], [10], [11]), by using spectral theory,

$$
E_{2}(T) \ll T^{2 / 3} \log ^{C_{1}} T,
$$

$$
\int_{0}^{T} E_{2}^{2}(t) d t \ll T^{2} \log ^{C_{2}} T, \quad \int_{0}^{T} E_{2}(t) d t \ll T^{3 / 2}
$$

with effective constants $C_{1}, C_{2}>0$. In [6] and [8] the first author has applied (1.12) with $k=2$ to obtain the following results: There exist constants $A, B>0$ such that for $T \geq T_{0}>0$ every interval $[T, A T]$ contains points $t_{1}, t_{2}, t_{3}, t_{4}$ such that

$$
\begin{aligned}
E_{2}\left(t_{1}\right)>B t_{1}^{1 / 2}, & E_{2}\left(t_{2}\right)<-B t_{2}^{1 / 2}, \\
\int_{0}^{t_{3}} E_{2}(t) d t>B t_{3}^{3 / 2}, & \int_{0}^{t_{4}} E_{2}(t) d t<-B t_{4}^{3 / 2} .
\end{aligned}
$$

Moreover, we have

$$
\int_{0}^{T} E_{2}^{2}(t) d t \gg T^{2}
$$

which complements the upper bound in (1.13). For other relevant recent work on the fourth moment of $|\zeta(1 / 2+i t)|$, all of which is based on the use of powerful methods from spectral theory, the reader is referred to [13], [14], [18]-[20] and [22]. For the proof of (1.12) it was necessary to have an order result for $\mathcal{Z}_{2}(s)$ in $\sigma>\varepsilon-1 / 2$ for any given $\varepsilon>0$, and the following weak result (see [8]) was sufficient: For any given $\eta>0$ we have $\mathcal{Z}_{2}(s) \ll e^{\eta|\operatorname{Im} s|}$, provided that $s$ stays away from the $\varepsilon$-neighbourhoods of poles of $\mathcal{Z}_{2}(s)$. However, for other possible applications it seems desirable to have a stronger result on the growth of $\mathcal{Z}_{2}(s)$. Namely the bound $\mathcal{Z}_{2}(s) \ll e^{\eta|\operatorname{Im} s|}$ should be replaced by a bound which gives polynomial growth in $|\operatorname{Im} s|$, that is $\mathcal{Z}_{2}(s) \ll|\operatorname{Im} s|^{C(\sigma)}$ with a suitable constant $C(\sigma)>0$, again provided that $s$ stays away from the $\varepsilon$-neighbourhoods of poles of $\mathcal{Z}_{2}(s)$. This order result, whose proof is long and difficult, is the central theme of this paper and it is 
given as Theorem 3 in Section 4. In Section 5 we shall obtain pointwise and mean-square bounds for $\mathcal{Z}_{2}(s)$ in the region $1 / 2<\sigma<1$ that are better than those which follow from Theorem 3.

2. A bound for the eighth moment. At the beginning we mentioned the possibility of using (1.1) for mean square estimates. Having in mind that we now possess quite a lot of information on $\mathcal{Z}_{2}(s)$ and very little on $\mathcal{Z}_{k}(s)$ when $k>2$, it is natural to ask whether we could put our knowledge on $\mathcal{Z}_{2}(s)$ to advantage and treat higher power moments of $|\zeta(1 / 2+i t)|$ with it. This is certainly possible, especially the eighth moment seems the natural one to attack by trying to connect it with $\mathcal{Z}_{2}(s)$, and thus indirectly with bounds from spectral theory. On the other hand, the technical difficulties involved in executing this program appear to be overwhelming, and the bounds that we have managed to obtain are rather complicated and do not seem to give any improvement yet of (1.5) when $k=4$. But the approach is, in principle, of interest, and we propose to give here a result which connects the eighth moment of $|\zeta(1 / 2+i t)|$ with the mean square of the Mellin transform of $E_{2}(x)$. Let

$$
\mathcal{G}(s)=\int_{0}^{\infty} \varphi(x) E_{2}(x) x^{s-1} d x \quad(s=\sigma+i t),
$$

where $\varphi \in C^{\infty}(0, \infty)$ is a test function such that $\varphi(x) \geq 0, \varphi(x)=1$ for $T \leq x \leq 2 T, \varphi(x)=0$ for $x<T / 2$ or $x>5 T / 2\left(T \geq T_{0}>0\right), \varphi(x)$ is increasing in $[T / 2, T]$ and decreasing in $[2 T, 5 T / 2]$. Then we have

TheOREM 1. There is a constant $C>0$ such that

$$
\begin{aligned}
\int_{0}^{\infty} \varphi^{2}(x) x^{2}|\zeta(1 / 2+i x)|^{8} d x & \\
= & \frac{1}{\pi} \int_{T^{1 / 2}}^{T^{1+\varepsilon}} t^{2}|\mathcal{G}(1 / 2+i t)|^{2} d t+O\left(T^{3} \log C\right) .
\end{aligned}
$$

Corollary 1. There is a constant $C>0$ such that

$$
\int_{T}^{2 T}|\zeta(1 / 2+i t)|^{8} d t \ll_{\varepsilon} T^{-2} \int_{T^{1 / 2}}^{T^{1+\varepsilon}} t^{2}|\mathcal{G}(1 / 2+i t)|^{2} d t+T \log ^{C} T .
$$

Before giving a proof of Theorem 1 we shall show how to connect $\mathcal{Z}_{2}(s)$ and $\mathcal{G}(s)$, so that $(2.3)$ can be used as a starting point to estimate the eighth moment of $|\zeta(1 / 2+i t)|$ by a mean square bound which can be reduced to bounds from spectral theory. From (1.10) with $k=2$ and $Q_{4}=P_{4}+P_{4}^{\prime}$ we 
have, for $\operatorname{Re} w>1$,

$$
\begin{aligned}
\mathcal{Z}_{2}(w) & =\int_{1}^{\infty} x^{-w} d\left(\int_{0}^{x}|\zeta(1 / 2+i t)|^{4} d t\right) \\
& =\int_{1}^{\infty} x^{-w} Q_{4}(\log x) d x+\int_{1}^{\infty} x^{-w} d E_{2}(x) \\
& =\sum_{j=0}^{5} c_{j}(w-1)^{-j}+w \int_{1}^{\infty} E_{2}(x) x^{-w-1} d x,
\end{aligned}
$$

say. The constants $c_{j}$ may be explicitly evaluated, and $\sum_{j=1}^{5} c_{j}(w-1)^{-j}$ represents in fact the principal part of the Laurent expansion of $\mathcal{Z}_{2}(w)$ at $w=1$. The last integral in (2.4) is, in view of the mean square bound in (1.13), a regular function of $w$ for $\operatorname{Re} w>1 / 2$. Thus (2.4) yields analytic continuation of $\mathcal{Z}_{2}(w)$ to the half-plane $\operatorname{Re} w>1 / 2$, but it is the region $\operatorname{Re} w \leq 1 / 2$ which is of course more difficult and more interesting. Now let $\varphi(t)=\varphi_{T}(t)=\Phi(t / T)$, where $\Phi(x)$ is a smooth function supported in $[1 / 2,5 / 2]$ such that $\Phi(x)=1$ for $1 \leq x \leq 2, \Phi(x)$ is increasing in $[1 / 2,1]$ and decreasing in $[2,5 / 2]$. Let further

$$
\mathcal{H}(w)=\mathcal{H}_{s, T}(w):=T^{s-1} \int_{0}^{\infty} \Phi(x) x^{s-1} \cdot x^{w-1} d x
$$

be the Mellin transform of $(T x)^{s-1} \Phi(x)$ for a given $T(\gg 1)$ and $s \in \mathbb{C}$ (it is the imaginary part of $s$ that is relevant in subsequent considerations, while actually the value of $\sigma=\operatorname{Re} s$ does not matter). Then by the Mellin inversion formula we have, for any $d \in \mathbb{R}$,

$$
(T x)^{s-1} \Phi(x)=\frac{1}{2 \pi i} \int_{(d)} \mathcal{H}(z) x^{-z} d z .
$$

To obtain a suitable representation of $\mathcal{G}(s)$ in terms of $\mathcal{Z}_{2}(s)$ we consider the integral

$$
I:=\frac{1}{2 \pi i} \int_{(a)} \mathcal{Z}_{2}(w) T^{w+1} \mathcal{H}(w+1) \frac{d w}{w} \quad(a>1) .
$$

We obtain from (2.4)-(2.6) and absolute convergence, for $a>1$,

$$
\begin{aligned}
I= & \frac{1}{2 \pi i} \int_{(a)}\left(\sum_{j=0}^{5} c_{j}(w-1)^{-j}\right) T^{w+1} \mathcal{H}(w+1) \frac{d w}{w} \\
& +\int_{1}^{\infty} E_{2}(x)\left(\frac{1}{2 \pi i} \int_{(a)} \mathcal{H}(w+1)\left(\frac{x}{T}\right)^{-w-1} d w\right) d x
\end{aligned}
$$




$$
\begin{aligned}
= & \frac{1}{2 \pi i} \int_{(a)}\left(\sum_{j=0}^{5} c_{j}(w-1)^{-j}\right) T^{w+1} \mathcal{H}(w+1) \frac{d w}{w} \\
& +\int_{1}^{\infty} E_{2}(x) x^{s-1} \Phi\left(\frac{x}{T}\right) d x \\
= & \frac{1}{2 \pi i} \int_{(a)}\left(\sum_{j=0}^{5} c_{j}(w-1)^{-j}\right) T^{w+1} \mathcal{H}(w+1) \frac{d w}{w}+\mathcal{G}(s) .
\end{aligned}
$$

Therefore for $a>1$ we may write

$$
\mathcal{G}(s)=\frac{1}{2 \pi i} \int_{(a)}\left(\mathcal{Z}_{2}(w)-\sum_{j=0}^{5} c_{j}(w-1)^{-j}\right) T^{w+s} U(s, w) \frac{d w}{w},
$$

where

$$
U(s, w):=\int_{0}^{\infty} \Phi(x) x^{s+w-1} d x=\int_{1 / 2}^{5 / 2} \Phi(x) x^{s+w-1} d x .
$$

This is an entire function of $s$ and $w$, and to truncate integrals containing $U(s, w)$ we may use the bound, valid for any fixed $A>0$ and bounded $\operatorname{Re}(s+w)$,

$$
U(s, w) \ll \min \left(1,\left|\frac{s}{w}\right|^{A},\left|\frac{w}{s}\right|^{A}, \frac{1}{|s+w|^{A}}\right) .
$$

Proof of Theorem 1. Let

$$
\begin{aligned}
\mathcal{F}(s) & :=\int_{0}^{\infty} \varphi(x)|\zeta(1 / 2+i x)|^{4} x^{s-1} d x \\
& =\int_{T / 2}^{5 T / 2} \varphi(x)|\zeta(1 / 2+i x)|^{4} x^{s-1} d x
\end{aligned}
$$

be the Mellin transform of $\varphi(x)|\zeta(1 / 2+i x)|^{4}$. The function $\mathcal{F}(s)$ is entire and of rapid decay in any fixed vertical strip and (1.1) gives

$$
\int_{0}^{\infty} \varphi^{2}(x)|\zeta(1 / 2+i x)|^{8} x^{2 \sigma-1} d x=\frac{1}{\pi} \int_{0}^{\infty}|\mathcal{F}(\sigma+i t)|^{2} d t .
$$

To truncate the integral on the right-hand side of (2.11), let as usual

$$
Z(t):=\chi^{-1 / 2}(1 / 2+i t) \zeta(1 / 2+i t), \quad \zeta(s)=\chi(s) \zeta(1-s),
$$

so that $Z(t)$ is real-valued and $|Z(t)|=|\zeta(1 / 2+i t)|$. Performing $\nu$ integra- 
tions by parts we obtain, for $\nu \in \mathbb{N}$,

$$
\mathcal{F}(s)=\frac{(-1)^{\nu}}{s(s+1) \ldots(s+\nu-1)} \int_{T / 2}^{5 T / 2} x^{s+\nu-1}\left(\varphi(x) Z^{4}(x)\right)^{(\nu)} d x .
$$

By Leibniz's rule for the $\nu$ th derivative of a product we have

$$
\begin{aligned}
\left(\varphi(x) Z^{4}(x)\right)^{(\nu)} & =\sum_{l=0}^{\nu}\left(\begin{array}{l}
\nu \\
l
\end{array}\right) \varphi^{(l)}(x)\left(Z^{2}(x) Z^{2}(x)\right)^{(\nu-l)} \\
& \ll \sum_{l=0}^{\nu} T^{-l} \sum_{m=0}^{\nu-l}\left|\left(Z^{2}(x)\right)^{(m)}\left(Z^{2}(x)\right)^{(\nu-l-m)}\right|,
\end{aligned}
$$

since $\varphi^{(l)}(x) \ll_{l} T^{-l}(l=0,1, \ldots)$. We insert the above bound in $(2.11)$, use the Cauchy-Schwarz inequality and the fact that $\left(Z^{2}(x)\right)^{(m)}$ is bounded in mean square by a suitable log-power. This follows by Hölder's inequality for integrals, the fact that (see [15, Chapter 3])

$$
\begin{array}{r}
Z^{(k)}(t)=O_{k}\left(t^{-1 / 4}\left(\frac{3}{2} \log t\right)^{k+1}\right)+2 \sum_{n \leq \sqrt{t /(2 \pi)}} n^{-1 / 2}\left(\log \frac{\sqrt{t /(2 \pi)}}{n}\right)^{k} \\
\times \cos \left(t \log \frac{\sqrt{t /(2 \pi)}}{n}-\frac{t}{2}-\frac{\pi}{8}+\frac{\pi k}{2}\right),
\end{array}
$$

and the mean value theorem for Dirichlet polynomials. Another way to see this is to use Leibniz's formula for the derivative of a product, the expression for $\chi(s)$ and properties of the gamma-function.

We thus obtain

$$
\mathcal{F}(s) \ll_{\nu} t^{-\nu} T^{\sigma+\nu} \log ^{c(\nu)} T \quad\left(s=\sigma+i t, t \geq t_{0}>0, \sigma>0\right) .
$$

The bound (2.12) shows, on taking $\nu=\nu(\varepsilon)$ sufficiently large, that (2.11) becomes

$$
\int_{0}^{\infty} \varphi^{2}(x)|\zeta(1 / 2+i x)|^{8} x^{2 \sigma-1} d x=\frac{1}{\pi} \int_{0}^{T^{1+\varepsilon}}|\mathcal{F}(\sigma+i t)|^{2} d t+O_{\varepsilon}(1) .
$$

We can also write $\left(Q_{4}=P_{4}+P_{4}^{\prime}\right)$

$$
\begin{aligned}
\mathcal{F}(s) & =\int_{T / 2}^{5 T / 2} \varphi(x) x^{s-1} d\left(\int_{0}^{x}|\zeta(1 / 2+i t)|^{4} d t\right) \\
& =\int_{T / 2}^{5 T / 2} \varphi(x) x^{s-1}\left(Q_{4}(\log x) d x+d E_{2}(x)\right)
\end{aligned}
$$




$$
\begin{aligned}
= & \int_{T / 2}^{5 T / 2} \varphi(x) x^{s-1} Q_{4}(\log x) d x \\
& -\int_{T / 2}^{5 T / 2} E_{2}(x)\left(\varphi^{\prime}(x) x^{s-1}+(s-1) \varphi(x) x^{s-2}\right) d x \\
= & -\int_{T / 2}^{5 T / 2} \frac{x^{s}}{s}\left(\frac{\varphi(x)}{x} Q_{4}^{\prime}(\log x)+\varphi^{\prime}(x) Q_{4}(\log x)\right) d x \\
& -\int_{T / 2}^{5 T / 2} E_{2}(x) \varphi^{\prime}(x) x^{s-1} d x+(1-s) \mathcal{G}(s-1),
\end{aligned}
$$

where $\mathcal{G}(s)$ is defined by (2.1). Trivial estimation gives

$$
\mathcal{F}(s) \ll T^{\sigma} \log ^{4} T \quad\left(|t| \leq t_{0}\right),
$$

where $t_{0}$ is a (large) positive constant. Note that for $|t| \geq t_{0}$ we have (using $E_{2}(T) \ll T^{2 / 3} \log ^{C} T$ and the bound for the fourth moment)

$$
\begin{aligned}
\int_{T / 2}^{5 T / 2} E_{2}(x) \varphi^{\prime}(x) x^{s-1} d x & =-\int_{T / 2}^{5 T / 2} \frac{x^{s}}{s}\left(\varphi^{\prime \prime}(x) E_{2}(x)+\varphi^{\prime}(x) E_{2}^{\prime}(x)\right) d x \\
& \ll \frac{T^{\sigma} \log ^{4} T}{|t|} .
\end{aligned}
$$

If we integrate once more by parts the above integral we find that it is

$$
\ll T^{\sigma+1} t^{-2} \log ^{5} T,
$$

noting that

$$
E_{2}^{\prime \prime}(x)=-x^{-1}\left(P_{4}^{\prime}(\log x)+P_{4}^{\prime \prime}(\log x)\right)+4 Z^{3}(x) Z^{\prime}(x),
$$

using Hölder's inequality, and then trivial estimation. The same bound holds for the first integral in the last expression in (2.14). Therefore, for $t \geq t_{0}>0$, (2.14) gives

$$
\begin{gathered}
\mathcal{F}(s)=(1-s) \mathcal{G}(s-1)+R(s, T), \\
R(s, T) \ll \min \left(T^{\sigma} t^{-1} \log ^{4} T, T^{\sigma+1} t^{-2} \log ^{5} T\right) .
\end{gathered}
$$

From (2.13) and (2.15) we infer

$$
\begin{aligned}
\int_{0}^{\infty} \varphi^{2}(x)|\zeta(1 / 2+i x)|^{8} x^{2 \sigma-1} d x & \\
& =\frac{1}{\pi} \int_{t_{0}}^{T^{1+\varepsilon}}|\mathcal{F}(\sigma+i t)|^{2} d t+O_{\varepsilon}\left(T^{2 \sigma} \log ^{8} T\right) .
\end{aligned}
$$


Inserting (2.16) in (2.17) we obtain

$$
\begin{aligned}
\int_{0}^{\infty} \varphi^{2}(x) \mid \zeta & \left.(1 / 2+i x)\right|^{8} x^{2 \sigma-1} d x \\
= & \frac{1}{\pi} \int_{t_{0}}^{T^{1+\varepsilon}}|(1-s) \mathcal{G}(s-1)|^{2} d t \\
& +O\left(T^{2 \sigma} \log ^{8} T\right)+O\left(\int_{t_{0}}^{T^{1+\varepsilon}} t|R(\sigma+i t, T) \mathcal{G}(\sigma-1+i t)| d t\right),
\end{aligned}
$$

which with $\sigma=3 / 2$ becomes (splitting the last integral above at $t=T$ and using the appropriate bound for $R(s, T)$ in each range)

$$
\begin{aligned}
\int_{0}^{\infty} \varphi^{2}(x) \mid \zeta & \left.(1 / 2+i x)\right|^{8} x^{2} d x \\
= & \frac{1}{\pi} \int_{t_{0}}^{T^{1+\varepsilon}} t^{2}|\mathcal{G}(1 / 2+i t)|^{2} d t+O\left(\int_{t_{0}}^{T^{1+\varepsilon}}|\mathcal{G}(1 / 2+i t)|^{2} d t\right) \\
& +O\left(T^{3} \log ^{8} T\right)+O\left(T^{2} \log ^{5} T\left(\int_{t_{0}}^{T^{1+\varepsilon}}|\mathcal{G}(1 / 2+i t)|^{2} d t\right)^{1 / 2}\right) .
\end{aligned}
$$

If we use (1.1) with $F(s)=G(s)=\mathcal{G}(s)$, then similarly to (2.13) we obtain

$$
\int_{0}^{\infty} \varphi^{2}(x) E_{2}^{2}(x) x^{2 \sigma-1} d x=\frac{1}{\pi} \int_{0}^{T^{1+\varepsilon}}|\mathcal{G}(\sigma+i t)|^{2} d t+O_{\varepsilon}(1) .
$$

But from (2.18) with $\sigma=1 / 2$ and the mean-square bound

$$
\int_{0}^{T} E_{2}^{2}(t) d t \ll T^{2} \log ^{C} T
$$

we obtain

$$
\begin{aligned}
\frac{1}{\pi} \int_{0}^{T^{1+\varepsilon}}|\mathcal{G}(1 / 2+i t)|^{2} d t & =\int_{0}^{\infty} \varphi^{2}(x) E_{2}^{2}(x) d x+O_{\varepsilon}(1) \\
& \ll \int_{T / 2}^{5 T / 2} E_{2}^{2}(x) d x \ll T^{2} \log ^{C} T .
\end{aligned}
$$

Hence using (2.20) we have 


$$
\begin{aligned}
\int_{0}^{\infty} & \varphi^{2}(x)|\zeta(1 / 2+i x)|^{8} x^{2} d x \\
& =\frac{1}{\pi} \int_{t_{0}}^{T^{1+\varepsilon}} t^{2}|\mathcal{G}(1 / 2+i t)|^{2} d t+O\left(T^{3} \log ^{C} T\right) \\
& =\frac{1}{\pi} \int_{T^{1 / 2}}^{T^{1+\varepsilon}} t^{2}|\mathcal{G}(1 / 2+i t)|^{2} d t+O\left(\int_{t_{0}}^{T^{1 / 2}} t^{2}|\mathcal{G}(1 / 2+i t)|^{2} d t\right)+O\left(T^{3} \log ^{C} T\right) \\
& =\frac{1}{\pi} \int_{T^{1 / 2}}^{T^{1+\varepsilon}} t^{2}|\mathcal{G}(1 / 2+i t)|^{2} d t+O\left(T \int_{0}^{T^{1+\varepsilon}}|\mathcal{G}(1 / 2+i t)|^{2} d t\right)+O\left(T^{3} \log ^{C} T\right) \\
& =\frac{1}{\pi} \int_{T^{1 / 2}}^{T^{1+\varepsilon}} t^{2}|\mathcal{G}(1 / 2+i t)|^{2} d t+O\left(T^{3} \log ^{C} T\right),
\end{aligned}
$$

as asserted $(C=22$ will do, since with the value $C=22$ the bound (2.19) holds in view of $[24,(5.2 .21)])$. This completes the proof of Theorem 1.

It would be very interesting if in (2.2) and (2.3) the upper limit of integration $T^{1+\varepsilon}$ in the integral on the right-hand side could be replaced by a smaller one (with an error which is $\ll T^{1+\varepsilon}$ ). Suppose that instead of $(2.3)$ we had

$$
\int_{T}^{2 T}|\zeta(1 / 2+i t)|^{8} d t \ll_{\varepsilon} T^{-2} \int_{T^{1 / 2}}^{T^{\omega+\varepsilon}} t^{2}|\mathcal{G}(1 / 2+i t)|^{2} d t+T^{1+\varepsilon}
$$

for some $\omega$ satisfying $1 / 2<\omega<1$. Then from (2.20) it would follow that

$$
\int_{T}^{2 T}|\zeta(1 / 2+i t)|^{8} d t \ll_{\varepsilon} T^{2 \omega+\varepsilon},
$$

so that with $\omega=3 / 4$ we would recover from (2.22) the sharpest known bound (up to a log-factor) for the eighth moment. For a bound that complements (2.22), see (5.9).

3. The function $\mathcal{Z}_{1}(s)$. In this section we shall discuss the analytic behaviour of the function $\mathcal{Z}_{1}(s)$ (see (1.3)), obtain its analytic continuation to the half-plane $\sigma>-3 / 4$, and derive pointwise and mean square bounds in suitable strips. A natural tool to be used in connection with mean square problems involving $\zeta(s)$ is the well-known formula of F. V. Atkinson [1] for 
the function $E_{1}(T) \equiv E(T)$ in (1.10) (see also [4] and [5]). We have

$$
\int_{0}^{T}|\zeta(1 / 2+i t)|^{2} d t=T \log \left(\frac{T}{2 \pi}\right)+(2 \gamma-1) T+E(T),
$$

where $\gamma=0.577215 \ldots$ is Euler's constant, and we also have the mean square formula

$$
\int_{0}^{T} E^{2}(t) d t \sim C T^{3 / 2} \quad(C>0, T \rightarrow \infty) .
$$

Hence from (3.1) and (3.2) it follows that

$$
\begin{aligned}
\mathcal{Z}_{1}(s) & =\int_{1}^{\infty}(\log x+2 \gamma-\log (2 \pi)) x^{-s} d x+\int_{1}^{\infty} E^{\prime}(x) x^{-s} d x \\
& =\frac{1}{(s-1)^{2}}+\frac{2 \gamma-\log (2 \pi)}{s-1}-E(1)+s \int_{1}^{\infty} E(x) x^{-s-1} d x,
\end{aligned}
$$

where the last integral converges absolutely for $\sigma>1 / 4$. Thus we have

$$
\begin{array}{r}
\mathcal{Z}_{1}(s)=\frac{1}{(s-1)^{2}}+\frac{2 \gamma-\log (2 \pi)}{s-1}-E(1)+s \int_{1}^{\infty} E(x) x^{-s-1} d x \\
(\sigma>1 / 4) .
\end{array}
$$

Further analytic continuation (and polynomial growth in $|s|$ ) may be obtained by integration by parts of the integral in (3.3). To this end let

$$
G(T):=\int_{1}^{T} E(t) d t-\pi T, \quad G_{1}(T):=\int_{1}^{T} G(t) d t .
$$

Now we invoke a result of Hafner-Ivić [3] (see also [5]) which says that

$$
G(T)=S_{1}(T ; N)-S_{2}(T ; N)+O\left(T^{1 / 4}\right)
$$

with

$$
\begin{aligned}
S_{1}(T ; N)= & 2^{-3 / 2} \sum_{n \leq N}(-1)^{n} d(n) n^{-1 / 2} \\
& \times\left(\operatorname{arsinh} \sqrt{\frac{\pi n}{2 T}}\right)^{-2}\left(\frac{T}{2 \pi n}+\frac{1}{4}\right)^{-1 / 4} \sin (f(T, n)), \\
S_{2}(T ; N)= & \sum_{n \leq N^{\prime}} d(n) n^{-1 / 2}\left(\log \frac{T}{2 \pi n}\right)^{-2} \sin (g(T, n)), \\
f(T, n)= & 2 T \operatorname{arsinh} \sqrt{\frac{\pi n}{2 T}}+\sqrt{2 \pi n T+\pi^{2} n^{2}}-\frac{\pi}{4},
\end{aligned}
$$




$$
g(T, n)=T \log \left(\frac{T}{2 \pi n}\right)-T+\frac{\pi}{4}, \quad \operatorname{arsinh} x=\log \left(x+\sqrt{x^{2}+1}\right),
$$

$A T<N<A^{\prime} T\left(0<A<A^{\prime}\right.$ constants $), \quad N^{\prime}=\frac{T}{2 \pi}+\frac{N}{2}-\sqrt{\frac{N^{2}}{4}+\frac{N T}{2 \pi}}$.

We use Taylor's formula (see [5, Lemma 3.2] and [3]) to simplify (3.5). Then we obtain

$$
\begin{aligned}
G(T)= & 2^{-1 / 4} \pi^{-3 / 4} T^{3 / 4} \sum_{n=1}^{\infty}(-1)^{n} d(n) n^{-5 / 4} \sin (\sqrt{8 \pi n T}-\pi / 4) \\
& +O\left(T^{2 / 3} \log T\right)
\end{aligned}
$$

so that

$$
G(T)=O\left(T^{3 / 4}\right), \quad G(T)=\Omega_{ \pm}\left(T^{3 / 4}\right) .
$$

We use (3.5) with $T=t, N=T, T \leq t \leq 2 T$ and apply the first derivative test ([4, Lemma 2.1]) to deduce that

$$
\int_{T}^{2 T} G(t) d t \ll T^{5 / 4} .
$$

Hence

$$
G_{1}(T)=\int_{1}^{T} G(t) d t \ll T^{5 / 4} .
$$

We have (for $\sigma>1 / 4$ )

$$
\begin{aligned}
\int_{1}^{\infty} E(x) x^{-s-1} d x & =(s+1) \int_{1}^{\infty} \int_{1}^{x} E(u) d u \cdot x^{-s-2} d x \\
& =(s+1) \int_{1}^{\infty}\left(\pi x^{-s-1}+G(x) x^{-s-2}\right) d x \\
& =\left(1+s^{-1}\right) \pi+(s+1) \int_{1}^{\infty} G(x) x^{-s-2} d x \\
& =\left(1+s^{-1}\right) \pi+(s+1)(s+2) \int_{1}^{\infty} G_{1}(x) x^{-s-3} d x .
\end{aligned}
$$

In view of (3.6) the last integral above is absolutely convergent for $\sigma>-3 / 4$. Therefore we obtain

$$
\begin{aligned}
\mathcal{Z}_{1}(s)= & \frac{1}{(s-1)^{2}}+\frac{2 \gamma-\log (2 \pi)}{s-1}-E(1)+\pi(s+1) \\
& +s(s+1)(s+2) \int_{1}^{\infty} G_{1}(x) x^{-s-3} d x \quad(\sigma>-3 / 4)
\end{aligned}
$$


as the analytic continuation of $\mathcal{Z}_{1}(s)$ to the half-plane $\sigma>-3 / 4$. Presumably further integrations by parts would yield meromorphic continuation of $\mathcal{Z}_{1}(s)$ to $\mathbb{C}$. To achieve this one would have to know the asymptotic behaviour of $G_{n}(T):=\int_{1}^{T} G_{n-1}(t) d t(n>1)$, which already for $n=2$ entails considerable technical complications. Thus we shall content ourselves with (3.7).

Another (but not unrelated) approach to the analytic continuation of $\mathcal{Z}_{1}(s)$ is to use Theorem 4.1 of [24]. This general result, with the choice

$$
g(x)=\frac{1}{2}\left(x^{2}+D^{2}\right)^{-s / 2} \quad(D>0),
$$

where $s$ is a sufficiently large positive number and the parameter $D$ is also taken sufficiently large, gives an explicit formula for the function

$$
\frac{1}{2} \int_{-\infty}^{\infty}\left(x^{2}+D^{2}\right)^{-s / 2}|\zeta(1 / 2+i x)|^{2} d x
$$

closely resembling $\mathcal{Z}_{1}(s)$. By analytic continuation, this formula can be extended to complex values of $s$, and then it yields meromorphic continuation (and polynomial growth in $|s|$ ) of $\mathcal{Z}_{1}(s)$ to $\mathbb{C}$; the function $\mathcal{Z}_{1}(s)$ has a double pole at $s=1$ and other poles at $s=-n, n \in \mathbb{N}$. This approach may be compared to the way one obtained meromorphic continuation of $\mathcal{Z}_{2}(s)$ to $\mathbb{C}$ in [24, Section 5.3]. Without going into details of this argument, we briefly compare it with that based on formulae of the Atkinson type.

As an indication of the similarity between these two approaches, the alternative one leads to exponential integrals analogous to those occurring in the proof of Atkinson's formula, and these can be approximately evaluated by the saddle point method. However, by quoting Atkinson's formula (or its integrated version) as a known result, we shall avoid such calculations, and consequently the argument becomes more straightforward. It is a price to be paid for this simplification that the existence of error terms limits the analytic continuation to a half-plane only, instead of the whole plane. On the other hand, the alternative, more "uncompromised", argument is perfectly explicit with no error terms, and therefore it seems to be of wider scope, though admittedly it is technically somewhat more involved. To avoid complications, we prefer to proceed with our analysis of the function $\mathcal{Z}_{1}(s)$ choosing its representation (3.3) as the starting point in the proof of the following theorem.

TheOREM 2. If $G_{1}(T)$ is defined by (3.4), then $\mathcal{Z}_{1}(s)$ is regular for $\sigma>$ $-3 / 4$, except for a pole of order two at $s=1$, and

$$
\begin{aligned}
\mathcal{Z}_{1}(s)= & \frac{1}{(s-1)^{2}}+\frac{2 \gamma-\log (2 \pi)}{s-1}-E(1)+\pi(s+1) \\
& +s(s+1)(s+2) \int_{1}^{\infty} G_{1}(x) x^{-s-3} d x \quad(\sigma>-3 / 4) .
\end{aligned}
$$


Moreover, for $0 \leq \sigma \leq 1, t \geq t_{0}>0$, and $T \geq 1$, we have

$$
\mathcal{Z}_{1}(\sigma+i t) \ll_{\varepsilon} t^{1-\sigma+\varepsilon},
$$

and

$$
\int_{1}^{T}\left|\mathcal{Z}_{1}(\sigma+i t)\right|^{2} d t \ll_{\varepsilon} \begin{cases}T^{3-4 \sigma+\varepsilon} & (0 \leq \sigma \leq 1 / 2), \\ T^{2-2 \sigma+\varepsilon} & (1 / 2 \leq \sigma \leq 1) .\end{cases}
$$

P r o of. The first assertion was already stated in (3.7), so it remains to prove the pointwise and mean square estimates. We shall show that for the term

$$
Y(s):=s \int_{1}^{\infty} E(x) x^{-s-1} d x
$$

in (3.3) we have

$$
Y(\sigma+i t) \ll_{\varepsilon} T^{1-\sigma+\varepsilon} \quad(t \asymp T)
$$

and

$$
\int_{T}^{2 T}|Y(\sigma+i t)|^{2} d t \ll_{\varepsilon} T^{3-4 \sigma+\varepsilon} \quad(0 \leq \sigma \leq 1 / 2),
$$

from which (3.8) and the first bound in (3.9) follow. Although (3.3) is valid for $\sigma>1 / 4$, the arguments that follow will show that the range $\sigma>0$ may be treated. Actually, by convexity arguments, we may suppose that $\sigma \geq \sigma_{0}$ for some small positive constant $\sigma_{0}$, for $Y(s)$ is known to be holomorphic and of polynomial growth even until the line $\sigma=-1 / 2$, say.

We split up the integral in the definition of $Y(s)$ in subintegrals over $[X, 2 X]$, so that $Y(s)$ will be the sum of the entire functions

$$
Y_{X}(s):=s \int_{X}^{2 X} E(x) x^{-s-1} d x .
$$

Consider separately three cases: $X<T, T \leq X \leq T^{2}$, and $X>T^{2}$. In the first case, the assertions (3.10) and (3.11) for $Y_{X}(s)$ in place of $Y(s)$ are verified as follows. We apply integration by parts in (3.12), use the bound $E(x) \ll x^{1 / 3}$ (see [4, Chapter 15]) and estimate the mean square by Parseval's formula (1.1). Namely we use (1.1) with $1-\sigma$ in place of $\sigma$ and set

$$
f(x)=g(x)= \begin{cases}|\zeta(1 / 2+i x)|^{2}, & X \leq x \leq 2 X, \\ 0, & \text { otherwise }\end{cases}
$$

Then $F(s)=G(s)=\int_{X}^{2 X}|\zeta(1 / 2+i x)|^{2} x^{s-1} d x$, and we obtain

$$
\frac{1}{2 \pi} \int_{-\infty}^{\infty}|F(1-\sigma-i t)|^{2} d t=\int_{X}^{2 X}|\zeta(1 / 2+i x)|^{4} x^{1-2 \sigma} d x,
$$


which gives

$$
\begin{aligned}
\left.\left.\int_{T}^{2 T}\left|\int_{X}^{2 X}\right| \zeta(1 / 2+i x)\right|^{2} x^{-s} d x\right|^{2} d t & \ll \int_{X}^{2 X}|\zeta(1 / 2+i x)|^{4} x^{1-2 \sigma} d x \\
& \ll X^{2-2 \sigma} \log ^{4} X \ll T^{2-2 \sigma} \log ^{4} T .
\end{aligned}
$$

In the case $X>T$ we invoke Atkinson's formula for $E(T)$, which in the notation of $[4$, Theorem 15.1$]$ reads as follows:

$$
E(x)=\Sigma_{1}(x)+\Sigma_{2}(x)+O\left(\log ^{2} X\right) \quad(X \leq x \leq 2 X),
$$

and we choose $N=c X$ with $0<c<1$ a small constant. Here the term $\Sigma_{2}(x)$ is of logarithmic order in a mean square sense (cf. [4, (15.61)]), so its contribution together with that of the error term can be treated easily as above.

The contribution of $\Sigma_{1}(x)$ to $Y_{X}(s)$ is (all notation is as in [4, Theorem 15.1]) a constant multiple of

$$
s \sum_{n \leq c X}(-1)^{n} d(n) n^{-3 / 4} \int_{X}^{2 X} x^{-3 / 4-\sigma} e(x, n) e^{i F_{ \pm}(x)} d x,
$$

where

$$
\begin{gathered}
e(x, n):=\left(1+\frac{\pi n}{2 x}\right)^{-1 / 4}\left(\sqrt{\frac{2 x}{\pi n}} \operatorname{arsinh} \sqrt{\frac{\pi n}{2 x}}\right)^{-1}=1+O\left(\frac{n}{x}\right), \\
F_{ \pm}(x)=f(x, n) \pm t \log x,
\end{gathered}
$$

and $f(x, n)$ is as in (3.5). We may consider only $F:=F_{-}$, since $F_{+}$will have no saddle points, and its contribution is easily handled by the first derivative test (cf. [4, Lemma 2.1]). Note that

$$
F^{\prime}(x)=2 \log \left(\sqrt{\frac{\pi n}{2 x}}+\sqrt{1+\frac{\pi n}{2 x}}\right) \pm \frac{t}{x} \gg \sqrt{\frac{n}{X}}
$$

for $X \gg t^{2}$ and sufficiently small $c$. Hence by the first derivative test the contribution of such $X$ is

$$
\ll T \sum_{n \leq c X} d(n) n^{-3 / 4} X^{-3 / 4-\sigma} n^{-1 / 2} X^{1 / 2} \ll T X^{-1 / 4-\sigma} .
$$

Thus the series over the "dyadic" values $X>T^{2}$ converges, giving a contribution $\ll T^{1 / 2-2 \sigma}$ to $Y(s)$, and the contribution to the mean square is $\ll T^{2-4 \sigma}$.

Finally let $T \leq X \leq T^{2}$. If $n \geq c_{1} T^{2} / X$ with $c_{1}$ sufficiently large, then again $F^{\prime}(x) \gg \sqrt{n / X}$, and the preceding estimations apply to show that the corresponding sum over $n$ is $\ll T^{1 / 2} X^{-\sigma} \ll T^{1 / 2-\sigma}$, which suffices for our purposes. The first derivative test applies even if $n \leq c_{2} T^{2} / X$ with $c_{2}$ a sufficiently small positive constant; in this case we have $\left|F^{\prime}(x)\right| \gg T / X$. 
The critical range for $n$ is $n \asymp T^{2} / X$, when a saddle point for the integral in $Y_{X}(s)$ may occur, and this saddle point will be of the order $t^{2} / n$. And if there is a point $x_{0} \in[X, 2 X]$ such that $F^{\prime}\left(x_{0}\right)$ vanishes or at least attains a value which is small in comparison with $T / X$, then $\left|F^{\prime \prime}(x)\right| \asymp T / X^{2}$ near $x_{0}$, and elsewhere in the interval $[X, 2 X]$ we have $\left|F^{\prime}(x)\right| \asymp T / X$. To see this, note that $n / X$ is small, so the logarithmic term in the above formula for $F^{\prime}(x)$ behaves in the first approximation like the function $(2 \pi n / x)^{1 / 2}$. Thus, if the first derivative test applies, the argument is as above, and otherwise for $n \asymp T^{2} / X$ we use the second derivative test (cf. (2.5) of [4, Lemma 2.2]) to obtain a contribution to $Y_{X}(s)$ which is

$$
\ll T \sum_{n \asymp T^{2} / X}\left(T / X^{2}\right)^{-1 / 2} d(n) n^{-3 / 4} X^{-3 / 4-\sigma} \ll T X^{-\sigma+\varepsilon} \ll T^{1-\sigma+\varepsilon},
$$

and this completes the proof of (3.10).

For a proof of (3.11), we still have to estimate the integral

$$
T^{2} \int_{T}^{2 T}\left|\int_{X}^{2 X} \widetilde{\Sigma}_{1}(x) x^{-\sigma-i t-1} d x\right|^{2} d t
$$

where $\widetilde{\Sigma}_{1}(x)$ stands for the critical part of $\Sigma_{1}(x)$. Arguing as in the proof of the mean square estimate (3.2) (cf. [4, Section 15.4]), we have

$$
\int_{X}^{2 X}\left|\widetilde{\Sigma}_{1}(x)\right|^{2} d x \ll X^{\varepsilon}\left(X+X^{3 / 2}\left(T^{2} / X\right)^{-1 / 2}\right) \ll X^{2} T^{-1+\varepsilon} .
$$

Hence, again by use of Parseval's formula, we see that the expression (3.13) is

$$
\ll T^{1+\varepsilon} X^{1-2 \sigma} \ll T^{3-4 \sigma+\varepsilon},
$$

as required for finishing the proof of (3.11).

To prove the second mean square bound in (3.9) we start from the representation

$$
\begin{aligned}
\mathcal{Z}_{1}(s)= & \int_{1}^{X}|\zeta(1 / 2+i x)|^{2} x^{-s} d x \\
& +\frac{X^{1-s}}{s-1}\left(\frac{1}{s-1}+\log X+2 \gamma-\log (2 \pi)\right) \\
& -E(X) X^{-s}+s \int_{X}^{\infty} E(x) x^{-s-1} d x .
\end{aligned}
$$

This is valid for $X>1, \sigma>1 / 4$, and follows analogously to the proof of 
(3.3). Thus for $1 / 2 \leq \sigma \leq 1$ we obtain from (3.15), using (3.2) and Parseval's formula, similarly to (3.13),

$$
\begin{aligned}
\int_{T}^{2 T}\left|\mathcal{Z}_{1}(s)\right|^{2} d t \ll & \left.\left.\int_{T}^{2 T}\left|\int_{1}^{X}\right| \zeta(1 / 2+i x)\right|^{2} x^{-s} d x\right|^{2} d t+T^{-1} X^{2-2 \sigma} \log ^{2} X \\
& +T X^{2 / 3-2 \sigma}+T^{2} \int_{T}^{2 T}\left|\int_{X}^{\infty} E(x) x^{-s-1} d x\right|^{2} d t \\
\ll & \int_{1}^{X}|\zeta(1 / 2+i x)|^{4} x^{1-2 \sigma} d x \\
& +T^{-1} X^{2-2 \sigma} \log ^{2} X+T X^{2 / 3-2 \sigma}+T^{2} \int_{X}^{\infty} E^{2}(x) x^{-1-2 \sigma} d x \\
\ll & X^{2-2 \sigma} \log ^{5} X+T^{-1} X^{2-2 \sigma} \log ^{2} X \\
& +T X^{2 / 3-2 \sigma}+T^{2} X^{1 / 2-2 \sigma} \\
\ll & T^{8(1-\sigma) / 3} \log ^{5} T
\end{aligned}
$$

with the choice $X=T^{4 / 3}$. The second mean square bound in (3.9) now follows from the first bound in (3.9) at $\sigma=1 / 2$, and the above bound at $\sigma=1$ by the convexity of mean values [4, Lemma 8.3].

REMARK. Note that by the functional equation for $\zeta(s)$ we have

$$
\int_{1}^{T}|\zeta(\sigma+i t)|^{4} d t \ll_{\varepsilon} T^{3-4 \sigma+\varepsilon} \quad(0 \leq \sigma \leq 1 / 2) .
$$

Therefore (3.9) and (3.16) show that the analogy between $\mathcal{Z}_{1}(s)$ and $\zeta^{2}(s)$ is perfect as far as mean square estimates are concerned in the range $0 \leq$ $\sigma \leq 1 / 2$, whereas the pointwise estimate (3.8) for $\sigma=1 / 2$ corresponds to the standard "convexity bound" for the zeta-function. The analogy with the classical bound $\zeta(1 / 2+i t) \ll t^{1 / 6+\varepsilon}$ would be $\mathcal{Z}_{1}(1 / 2+i t) \ll t^{1 / 3+\varepsilon}$, and this is indeed accessible. The critical point where the argument can be elaborated is the application of the second derivative test at the end of the proof of Theorem 2. If the saddle point method is applied here, then an exponential sum involving the divisor function arises, and its trivial estimate by absolute values yields (3.8). However, a non-trivial estimate with sufficient saving follows from the second author's work [12], and thus one does obtain the bound $\mathcal{Z}_{1}(1 / 2+i t) \ll t^{1 / 3+\varepsilon}$. On the other hand, the analogy between $\mathcal{Z}_{1}(s)$ and $\zeta^{2}(s)$ breaks down for $\sigma>1 / 2$, and the underlying reason why $\mathcal{Z}_{1}(s)$ is "better" behaved in this domain is the continuity of the function $E(t)$ in contrast with the discontinuity of $\Delta(x)$. 
4. Analytic continuation and estimation of $\mathcal{Z}_{2}(s)$. In this section we shall discuss the analytic continuation and polynomial growth of the function $\mathcal{Z}_{2}(s)$, defined by (1.3). As before we assume that $\varepsilon$ denotes arbitrarily small positive constants, which are not necessarily the same at each occurrence. We shall prove the following

Theorem 3. Let $s=\sigma+i t, \varepsilon-1 / 2 \leq \sigma \leq 1-\varepsilon$. Then there exists a positive constant $B=B(\sigma)$ such that

$$
\mathcal{Z}_{2}(s) \ll(1+|t|)^{B}
$$

provided that $s$ stays away from the $\varepsilon$-neighbourhoods of poles of $\mathcal{Z}_{2}(s)$.

Proof. The strategy of the proof is as follows. Let $\xi \in(0,1)$ be a constant, and introduce

$$
\psi(T)=\frac{1}{\sqrt{\pi} T^{\xi}} \int_{-\infty}^{\infty}|\zeta(1 / 2+i(T+t))|^{4} \exp \left(-\left(t / T^{\xi}\right)^{2}\right) d t
$$

as a substitute for $|\zeta(1 / 2+i T)|^{4}$. Then we put

$$
\begin{aligned}
\mathcal{Z}_{2}(s) & =\int_{1}^{\infty} \psi(T) T^{-s} d T+\int_{1}^{\infty}\left(|\zeta(1 / 2+i T)|^{4}-\psi(T)\right) T^{-s} d T \\
& =\mathcal{Z}_{21}(s)+\mathcal{Z}_{22}(s),
\end{aligned}
$$

say. We expect that the integral $\mathcal{Z}_{22}(s)$ will converge well. In fact an elementary reformulation of $\mathcal{Z}_{22}(s)$ will show that this function is analogous to $\mathcal{Z}_{2}(s+2-2 \xi)$. But the assertion (4.1) is known to hold for $\mathcal{Z}_{2}(s)$ in the half-plane $\sigma \geq 1 / 2+\varepsilon$ (via integration by parts and (2.11)), and the same argument then gives a similar estimate for $\mathcal{Z}_{22}(s)$ in the half-plane $\sigma \geq 2 \xi-3 / 2+\varepsilon$, hence for $\sigma \geq-1 / 2+\varepsilon$ if $\xi \leq 1 / 2$, which will settle the estimation of $\mathcal{Z}_{22}$-part. On the other hand, the part $\mathcal{Z}_{21}(s)$ is more delicate. The function $\psi(T)$, with arbitrary $\xi \in(0,1)$, is expressed by a spectral decomposition formula (see [20] and $[24,(5.1 .1)]$ ), and the Mellin transform $\mathcal{Z}_{21}(s)$ of $\psi(T)$ is decomposed accordingly. The most significant contribution comes from the discrete spectrum, which will require some technically involved estimations. An essential point to be observed here is that the terms in the spectral expansion of $\psi(T)$ have much smoother expressions than the corresponding terms for $\mathcal{Z}_{2}(s)$ quoted above, and this will considerably facilitate subsequent treatment.

By definition we have

$$
\begin{aligned}
\mathcal{Z}_{22}(s)=-\frac{1}{\sqrt{\pi}} \int_{-\infty}^{\infty} \int_{1}^{\infty} T^{-\xi}\left(|\zeta(1 / 2+i(T+u))|^{4}-|\zeta(1 / 2+i T)|^{4}\right) \\
\times \exp \left(-\left(u / T^{\xi}\right)^{2}\right) T^{-s} d T d u
\end{aligned}
$$


for $\sigma>1$. Changing variables via $u=\alpha T^{\xi} \log T$, this becomes

$$
\begin{aligned}
\mathcal{Z}_{22}(s)=-\frac{1}{\sqrt{\pi}} \int_{-\infty}^{\infty} \int_{1}^{\infty}(\mid \zeta(1 / 2+i( & \left.\left.\left.+\alpha T^{\xi} \log T\right)\right)\left.\right|^{4}-|\zeta(1 / 2+i T)|^{4}\right) \\
\times & \exp \left(-\alpha^{2} \log ^{2} T\right) T^{-s}(\log T) d T d \alpha .
\end{aligned}
$$

Here the contribution of $|\alpha| \geq b$ for any fixed $b>0$ is obviously an entire function $B(s)$, say, which is bounded in any fixed vertical strip. Thus we may restrict $\alpha$ henceforth to the interval $|\alpha| \leq b$ with $b$ a small positive constant. Differentiation of (1.10) (with $k=2)$ gives $|\zeta(1 / 2+i T)|^{4}=Q_{4}(\log T)+$ $E_{2}^{\prime}(T)$, where $Q_{4}=P_{4}+P_{4}^{\prime}$ is a polynomial of degree four. Hence we have

$$
\mathcal{Z}_{22}(s)=-\frac{1}{\sqrt{\pi}}\left(\mathcal{Z}_{22}^{*}(s)+\mathcal{Z}_{22}^{* *}(s)\right)+B(s),
$$

where

$$
\mathcal{Z}_{22}^{*}(s):=\int_{1}^{\infty} \int_{-b \log T}^{b \log T}\left(Q_{4}\left(\log \left(T+\alpha T^{\xi}\right)\right)-Q_{4}(\log T)\right) T^{-s} e^{-\alpha^{2}} d \alpha d T
$$

and

$$
\begin{aligned}
\mathcal{Z}_{22}^{* *}(s):=\int_{-b}^{b} \int_{1}^{\infty}\left(E _ { 2 } ^ { \prime } \left(T+\alpha T^{\xi}\right.\right. & \left.\log T)-E_{2}^{\prime}(T)\right) \\
& \times \exp \left(-\alpha^{2} \log ^{2} T\right) T^{-s}(\log T) d T d \alpha .
\end{aligned}
$$

Note that in $\mathcal{Z}_{22}^{*}(s)$ we replaced $\alpha \log T$ again by $\alpha$. This function is a linear combination of integrals

$$
\int_{1}^{\infty} \int_{-b \log T}^{b \log T}\left(\log ^{m}\left(T+\alpha T^{\xi}\right)-\log ^{m} T\right) e^{-\alpha^{2}} T^{-s} d \alpha d T
$$

for $m=1,2,3,4$. The portion of the $T$-integral for $T \geq 100$ is an entire function. In the remaining portion we approximate $\log (1+x)$ by a Taylor polynomial of degree $K$ plus an error term. The latter will furnish a function which is regular for $\sigma>2(K+1)(\xi-1)+1$, and is bounded on $\sigma \geq$ $2(K+1)(\xi-1)+1+\varepsilon$ for each $\varepsilon>0$. There remain integrals of the type

$$
\int_{100}^{\infty} \int_{-b \log T}^{b \log T}\left(\log ^{\mu} T\right) \alpha^{2 k} e^{-\alpha^{2}} T^{2 k(\xi-1)-s} d \alpha d T
$$

where $0 \leq \mu<m$ and $k \leq 2 K$; note that the terms involving odd powers of $\alpha$ cancel out. Clearly we may extend the $\alpha$-integration to $(-\infty, \infty)$, which gives a constant depending on $k$. An integration by parts then shows that the $T$-integral has poles at the points $1-2 k(1-\xi)$. Hence $\mathcal{Z}_{22}^{*}(s)$ is bounded in 
any half-plane $\sigma \geq \sigma_{0}$ (since $K$ may be arbitrarily large) from which certain $\varepsilon$-neighbourhoods of the poles are excluded.

To consider the function $\mathcal{Z}_{22}^{* *}(s)$ let us rewrite the $T$-integral in (4.3) on substituting the new variable $\tau=\tau(T, \alpha)=T+\alpha T^{\xi} \log T$. If $\alpha$ is sufficiently small, as we may suppose, then $\tau(T, \alpha)$ is increasing as a function of $T$ for $T \geq 1$, and there is a monotonic inverse function $T=T(\tau, \alpha)$. For $T=T(\tau, \alpha)$, we have $T-\tau \ll(\log \tau) \tau^{\xi}$, hence $T \asymp \tau$, and the implicit equation for $T$ shows that

$$
T(\tau, \alpha)=\tau-\alpha \tau^{\xi} \log \tau+O\left(\tau^{2 \xi-1} \log ^{2} \tau\right) .
$$

Also we have

$$
\begin{aligned}
\frac{\partial T(\tau, \alpha)}{\partial \tau} & =\left(1+\alpha T^{\xi-1}(1+\xi \log T)\right)^{-1} \\
& =1-\alpha \tau^{\xi-1}(1+\xi \log \tau)+O\left(\tau^{2 \xi-2} \log ^{2} \tau\right) .
\end{aligned}
$$

For given positive $\alpha$, we combine the contributions of $\alpha$ and $-\alpha$ in (4.3). In the respective integrals, we put $\tau=\tau(T, \pm \alpha)$, and in the integral involving $E_{2}^{\prime}(T)$ we simply change the notation $T$ to $\tau$. Then (4.3) becomes, after an elementary rearrangement,

$$
\mathcal{Z}_{22}^{* *}(s)=\int_{0}^{b} \int_{1}^{\infty} E_{2}^{\prime}(\tau) f(\tau, \alpha) d \tau d \alpha,
$$

where

$$
\begin{aligned}
f(\tau, \alpha) & =f_{1}(\tau, \alpha)-f_{2}(\tau, \alpha), \\
f_{1}(\tau, \alpha) & =g(T(\tau, \alpha), \alpha)+g(T(\tau,-\alpha), \alpha)-2 g(\tau, \alpha), \\
f_{2}(\tau, \alpha) & =h(T(\tau, \alpha), \alpha)+h(T(\tau,-\alpha),-\alpha), \\
g(u, \alpha) & =(\log u) \exp \left(-\alpha^{2} \log ^{2} u\right) u^{-s}, \\
h(u, \alpha) & =\frac{\alpha(\log u) \exp \left(-\alpha^{2} \log ^{2} u\right) u^{\xi-s-1}(1+\xi \log u)}{1+\alpha u^{\xi-1}(1+\xi \log u)} .
\end{aligned}
$$

We shall consider the function $\mathcal{Z}_{22}^{* *}(s)$ in a horizontal strip $|t|<V$. To this end we split up the integral in (4.6) into two parts corresponding to the ranges $1 \leq \tau \leq X$ and $\tau>X$, where $X$ will be chosen in a moment. The first mentioned range gives rise to an entire function of $s$ which is clearly of the order

$$
\ll \log X \int_{1}^{X}\left(|\zeta(1 / 2+i \tau)|^{4}+\log ^{4} \tau\right) \tau^{-\sigma} d \tau \ll\left(1+X^{1-\sigma}\right) X^{\varepsilon} .
$$

To deal with the remaining integral, we note the estimates

$$
f(\tau, \alpha) \ll \tau^{2 \xi-2-\sigma}\left(\log ^{2} \tau+V \log \tau+V^{2}\right) \log ^{3} \tau,
$$




$$
\frac{\partial f(\tau, \alpha)}{\partial \tau} \ll \tau^{2 \xi-3-\sigma} V \log ^{3} \tau\left(\log ^{2} \tau+V \log \tau+V^{2}\right) .
$$

To verify (4.8), use the second order Taylor expansion of the function $g(u, \alpha)$ and linear expansions of the functions $h(u, \pm \alpha)$ near the point $\tau$, and substitute $u=T(\tau, \pm \alpha)$ from (4.5). Analogously, to verify (4.9), use (4.5) and the Taylor expansions of order two or one for $\frac{\partial}{\partial u} g(u, \alpha)$ and $\frac{\partial}{\partial u} h(u, \alpha)$ at $u=\tau$. By (4.8) and the standard mean value estimate for $|\zeta(1 / 2+i t)|^{4}$, we find that the integral

$$
\int_{0}^{b} \int_{X}^{\infty} E_{2}^{\prime}(\tau) f(\tau, \alpha) d \tau d \alpha
$$

is a regular function of $s$ for $\sigma>2 \xi-1,|t|<V$, and it is of the order

$$
\ll V^{2} X^{2 \xi-1-\sigma+\varepsilon} \text {. }
$$

To minimize the sum of the right-hand sides of (4.7) and (4.11), we choose

$$
X=V^{1 /(1-\xi)} .
$$

Recalling also our discussion of $\mathcal{Z}_{22}^{*}(s)$, we thus obtain the estimate

$$
\mathcal{Z}_{22}(s) \ll(|t|+1)^{(1-\sigma) /(1-\xi)+\varepsilon} \quad \text { for } \sigma \geq 2 \xi-1+\varepsilon,
$$

with the understanding that certain neighbourhoods of the poles are excluded. In particular, if we choose $\xi$ slightly smaller than $1 / 2$, then the estimate (4.13) holds in some half-plane $\sigma>-\varepsilon$.

Another possibility to deal with the integral (4.10) is to integrate by parts over $\tau$ and to use (4.9) together with the mean value estimate (2.11) for $E_{2}(\tau)$. This argument gives the estimate

$$
\ll V^{3} X^{2 \xi-3 / 2-\sigma+\varepsilon} \text { for } \sigma \geq 2 \xi-3 / 2+\varepsilon .
$$

The optimal choice for $X$ is now

$$
X=V^{6 /(5-4 \xi)}
$$

instead of (4.12), and in place of (4.13) we have

$$
\mathcal{Z}_{22}(s) \ll(|t|+1)^{(6-6 \sigma) /(5-4 \xi)+\varepsilon} \quad \text { for } \sigma \geq 2 \xi-3 / 2+\varepsilon .
$$

The bound (4.14) is weaker than (4.13) for $\xi<1 / 2$, but it holds in a wider range of $\sigma$. We also remark that the poles $1-2 k(1-\xi)$ of $\mathcal{Z}_{22}^{*}(s)$ depend on the parameter $\xi$ irrelevant of the function $\mathcal{Z}_{2}(s)$, so it is to be expected (and we shall see) that these will be cancelled out against the same poles of $\mathcal{Z}_{21}(s)$.

Next we turn to the function $\mathcal{Z}_{21}(s)$. We first note that in the notation of $[24,(5.1 .1)]$, we have

$$
\psi(T)=I_{2, r}\left(T, T^{\xi}\right)+I_{2, d}\left(T, T^{\xi}\right)+I_{2, c}\left(T, T^{\xi}\right)+I_{2, h}\left(T, T^{\xi}\right) .
$$


Here $I_{2, r}$ is an explicit main term, the transform of which is easy to compute and gives the principal part of the Laurent expansion of $\mathcal{Z}_{2}(s)$ at $s=1$ (a polynomial of fifth order in $\left.(s-1)^{-1}\right)$, together with terms with poles at $1-2 k(1-\xi)$; these compensate the poles of $\mathcal{Z}_{22}(s)$ mentioned above.

We next treat the transform of $I_{2, d}$, and that of $I_{2, c}$ will be commented afterwards, whereas the term $I_{2, h}$ as such is negligibly small (see [24, Lemma 5.1]). We quote from [24, p. 186], the following formula:

$$
I_{2, d}\left(T, T^{\xi}\right)=\sum_{j=1}^{\infty} \alpha_{j} H_{j}^{3}(1 / 2) \Lambda\left(\kappa_{j} ; T, T^{\xi}\right),
$$

where

$$
\begin{aligned}
\Lambda\left(r ; T, T^{\xi}\right)= & \frac{1}{2} \operatorname{Re}\left[\left(1+\frac{i}{\sinh \pi r}\right) \Xi\left(i r ; T, T^{\xi}\right)\right. \\
& \left.+\left(1-\frac{i}{\sinh \pi r}\right) \Xi\left(-i r ; T, T^{\xi}\right)\right]
\end{aligned}
$$

with

$$
\begin{aligned}
= & \frac{\Gamma^{2}(1 / 2+i r)}{\Gamma(1+2 i r)} \int_{0}^{\infty}(1+y)^{-1 / 2+i T} y^{-1 / 2+i r} \\
& \times \exp \left(-\frac{1}{4} T^{2 \xi} \log ^{2}(1+y)\right) F(1 / 2+i r, 1 / 2+i r ; 1+2 i r ;-y) d y,
\end{aligned}
$$

where $F$ (see [2]) is the hypergeometric function. Then we claim that

$$
\int_{1}^{\infty} I_{2, d}\left(T, T^{\xi}\right) T^{-s} d T=\sum_{j=1}^{\infty} \alpha_{j} H_{j}^{3}(1 / 2) \int_{1}^{\infty} \Lambda\left(\kappa_{j} ; T, T^{\xi}\right) T^{-s} d T,
$$

provided at least that $\operatorname{Re} s=\sigma$ is sufficiently large. That is, it is enough to study the transforms of the functions $\Lambda\left(r ; T, T^{\xi}\right)$ or rather those of $\Xi\left(i r ; T, T^{\xi}\right)$. To verify the required absolute convergence on the right-hand side it suffices to note the bounds

$$
\Xi\left(i r ; T, T^{\xi}\right) \ll \begin{cases}1 & \text { if }|r| \leq T \log ^{2} T, \\ |r|^{-C} & \text { otherwise. }\end{cases}
$$

This holds uniformly for any fixed $\xi \in(0,1)$ and any fixed large $C>0$. The first bound in (4.20) is a trivial consequence of (4.18) if the hypergeometric function is represented by its Gaussian integral formula (see [24, (5.1.7)(5.1.8)]), and the second follows from [24, Lemma 5.2]. It should be stressed that the main reason for introducing $\psi(T)$ above is to have the identity (4.19). Namely if we worked directly with the spectral decomposition of $\mathcal{Z}_{2}(s)[24$, pp. 206-207], then we would have encountered a serious difficulty here. 
Thus we consider, for $r>0$,

$$
X_{r}(s):=\int_{1}^{\infty} \Xi\left(-i r ; T, T^{\xi}\right) T^{-s} d T .
$$

This term is actually more critical than the term with $\Xi\left(i r ; T, T^{\xi}\right)$, which in view of (4.17) and (4.18) will also appear. Namely if the sign of $r$ is changed in (4.21), then the saddle-point of the $y$-integral in (4.18) disappears, and the estimations become more straightforward.

To begin with, we suppose that $\sigma>1$ to be sure that the Mellin transforms under consideration make sense as holomorphic functions. Our goal is the meromorphic continuation of $X_{r}(s)$ to the half-plane $\sigma>-1 / 2$ while attaining, simultaneously, a rapid decay with respect to $r$ and the polynomial growth with respect to $t$.

We suppose that

$$
1 / 3 \leq \xi \leq 1 / 2
$$

(cf. $[24,(5.1 .39)])$. Of course smaller values of $\xi$ would represent a more interesting case because then $\mathcal{Z}_{21}(s)$ looks more and more like $\mathcal{Z}_{2}(s)$. However, the value $1 / 3$ appears to be a "transition point"; as $\xi$ decreases, the argument gets messier.

Now we observe that for a given $r$ the last integral can be restricted to the interval $[T(r), \infty)$ with small error, provided that

$$
T(r)=r^{1 /(1-\xi)}(\log r)^{-D}
$$

for a suitable constant $D$. Indeed, by [24, Lemma 5.2] we have

$$
\Xi\left(-i r ; T, T^{\xi}\right) \ll(r T)^{-C} \quad \text { for } r \geq 2 T^{1-\xi} \log ^{5} T
$$

and any fixed $C>0$, whence the integrals over $[1, T(r)]$ for $r=\kappa_{j}$ produce a function of $s$ which is bounded and holomorphic in our half-plane $\sigma>-1 / 2$. In what follows we shall encounter similar "negligible" functions which can be omitted in the course of the argument; their contribution to the transform of $I_{2, d}\left(T, T^{\xi}\right)$ will be bounded in the half-plane $\sigma \geq-1 / 2+\varepsilon$ if the $\varepsilon$ neighbourhoods of the poles $1 / 2 \pm i \kappa_{j}$ of $\mathcal{Z}_{2}(s)$ are excluded. Hence our problem has become the estimation of

$$
X_{r}^{*}(s):=\int_{T(r)}^{\infty} \Xi\left(-i r ; T, T^{\xi}\right) T^{-s} d T .
$$

To this end, we substitute $y=z / T$ in (4.18). Then we have 


$$
\begin{aligned}
X_{r}^{*}(s)= & \frac{\Gamma^{2}(1 / 2-i r)}{\Gamma(1-2 i r)} \int_{T(r)}^{\infty} \int_{0}^{\infty} z^{-1 / 2-i r}\left(1+\frac{z}{T}\right)^{-1 / 2+i T} \\
& \times \exp \left(-\frac{1}{4} T^{2 \xi} \log ^{2}\left(1+\frac{z}{T}\right)\right) \\
& \times F(1 / 2-i r, 1 / 2-i r ; 1-2 i r ;-z / T) T^{-1 / 2+i r-s} d z d T .
\end{aligned}
$$

The crucial step in the analytic continuation of $X_{r}^{*}(s)$ is the $T$-integration by parts with respect to the last factor $T^{-1 / 2+i r-s}$ (cf. [24, (5.3.11)]). The integrated terms yield a negligible function; the argument to prove this can obviously be omitted. The new integral is a function having a pole at $s=1 / 2+i r$, but we shall see in a moment that it is otherwise holomorphic for $\sigma>-1 / 2$.

The integral in question is

$$
-\frac{\Gamma^{2}(1 / 2-i r)}{\Gamma(1-2 i r)}(1 / 2+i r-s)^{-1} \int_{T(r)}^{\infty} M(r ; T) T^{1 / 2+i r-s} d T
$$

where

$$
\begin{aligned}
M(r ; T):=\int_{0}^{\infty} z^{-1 / 2-i r} \frac{\partial}{\partial T}[(1+ & \left.\frac{z}{T}\right)^{-1 / 2+i T} \exp \left(-\frac{1}{4} T^{2 \xi} \log ^{2}\left(1+\frac{z}{T}\right)\right) \\
& \times F(1 / 2-i r, 1 / 2-i r ; 1-2 i r ;-z / T)] d z .
\end{aligned}
$$

We transform the hypergeometric function here by the formula $[17,(9.6 .12)]$

$$
F(\alpha, \alpha ; 2 \alpha ; z)=\left(\frac{1+\sqrt{1-z}}{2}\right)^{-2 \alpha} F\left(\alpha, \frac{1}{2} ; \alpha+\frac{1}{2} ;\left(\frac{1-\sqrt{1-z}}{1+\sqrt{1-z}}\right)^{2}\right)
$$

thus

$$
\begin{aligned}
F(1 / 2-i r, 1 / 2- & i r ; 1-2 i r ;-z / T) \\
= & 2^{1-2 i r}(1+\sqrt{1+z / T})^{-1+2 i r} \\
& \times F\left(\frac{1}{2}-i r, \frac{1}{2} ; 1-i r ;\left(\frac{1-\sqrt{1+z / T}}{1+\sqrt{1+z / T}}\right)^{2}\right) .
\end{aligned}
$$

Consider separately the ranges $z / T \leq r^{-a}$ and $z / T>r^{-a}$ for a small positive constant $a$. In the latter range, the exponential factor in $M(r ; T)$ is very small in view of (4.23), so that the contribution of this range to $M(r ; T)$ is $\ll T^{-A}$ for a large positive $A$. Therefore the corresponding part of (4.25) is a bounded holomorphic function of $s$ in any fixed vertical strip with a neighbourhood of the pole $1 / 2+i$ r excluded, and analogously for the respective part of the function (4.19). 
Let now $z / T \leq r^{-a}$. The virtue of the transformation (4.26) is that the new hypergeometric series converges rapidly. Namely in the familiar series expansion

$$
F(\alpha, \beta ; \gamma ; z)=\sum_{k=0}^{\infty} \frac{(\alpha)_{k}(\beta)_{k}}{(\gamma)_{k} k !} z^{k} \quad(|z|<1)
$$

we can take a finite number of terms in such the way that the tails of the series will make a negligible contribution. Since in our case $\alpha=1 / 2-$ ir, $\beta=1 / 2, \gamma=1-i r$, each term will yield similar expressions, and each contribution will be smaller than the one coming from the preceding term. Therefore the most significant term in the above series expansion will be simply the leading term 1 , so it suffices to consider its contribution. Then the essential part of $M(r ; T)$, say $M^{*}(r ; T)$, takes the form

$$
\begin{aligned}
M^{*}(r ; T):= & 2^{1-2 i r} \int_{0}^{T r^{-a}} z^{-1 / 2-i r} \frac{\partial}{\partial T}\left[\left(1+\frac{z}{T}\right)^{-1 / 2+i T}\right. \\
& \left.\times \exp \left(-\frac{1}{4} T^{2 \xi} \log ^{2}\left(1+\frac{z}{T}\right)\right)\left(1+\sqrt{1+\frac{z}{T}}\right)^{-1+2 i r}\right] d z .
\end{aligned}
$$

This integral can be approximately evaluated by the saddle point method. The exponential factor in $M^{*}(r ; T)$ is $e^{i F(z)}$ with

$$
F(z)=F(z ; r, T)=-r \log z+T \log \left(1+\frac{z}{T}\right)+2 r \log \left(1+\sqrt{1+\frac{z}{T}}\right),
$$

so that

$$
\begin{aligned}
& F^{\prime}(z)=-\frac{r}{z}+\frac{T}{T+z}+\frac{r}{T(\sqrt{1+z / T}+1+z / T)}, \\
& F^{\prime \prime}(z)=\frac{r}{z^{2}}-\frac{T}{(T+z)^{2}}-\frac{r(1+1 /(2 \sqrt{1+z / T}))}{T^{2}(\sqrt{1+z / T}+1+z / T)^{2}} .
\end{aligned}
$$

The saddle point $z_{0}$ is the solution of $F^{\prime}(z)=0$. We obtain

$$
\frac{z_{0}}{r}=\left(1+\frac{z_{0}}{T}\right)\left(1+\frac{r / T}{1+\left(1+z_{0} / T\right)^{-1 / 2}}\right)^{-1}
$$

which in view of $z_{0} / T \leq r^{-a}$ gives

$$
z_{0}=r+O\left(r^{2} / T\right)
$$

Since $T \geq T(r) \gg r^{3 / 2-\varepsilon}$ by (4.22) and (4.23), we also have $z_{0}=r+$ $O\left(r^{1 / 2+\varepsilon}\right)$. Then by a little calculation it is seen that $\left|F^{\prime \prime}\left(z_{0}\right)\right| \gg 1 / r$. On 
the other hand we have, in (4.27),

$$
\begin{aligned}
\frac{\partial}{\partial T}[\ldots]= & {[\ldots]\left\{i \log \left(1+\frac{z}{T}\right)+\frac{z}{T^{2}} \cdot \frac{1 / 2-i T}{1+z / T}\right.} \\
& +\left(1+\sqrt{1+\frac{z}{T}}\right)^{-1} \frac{(1-2 i r) z}{2 T^{2} \sqrt{1+z / T}} \\
& \left.-\frac{1}{2} \xi T^{2 \xi-1} \log ^{2}\left(1+\frac{z}{T}\right)+\frac{1}{2} T^{2 \xi} \log \left(1+\frac{z}{T}\right) \frac{z}{T^{2}}\right\} .
\end{aligned}
$$

We use the Taylor formula to find that, for $z=z_{0}=r+O\left(r^{2} / T\right)$, the expression in braces equals

$$
\begin{aligned}
\frac{i z_{0}}{2 T^{2}}\left(z_{0}-r\right)+\frac{3 z_{0}}{4 T^{2}} & +O\left(\frac{r^{3}}{T^{3}}\right)+O\left(r^{2} T^{2 \xi-3}\right) \\
& \ll r^{3} T^{-3}+r T^{-2}+r^{2} T^{2 \xi-3} \ll r T^{-2}+r^{2+\varepsilon} T^{2 \xi-3},
\end{aligned}
$$

since $1 / 3 \leq \xi \leq 1 / 2$ and (4.23) holds. Thus the contribution of the saddle point $z_{0}$ to $M^{*}(r ; T)$ is

$$
\ll\left|F^{\prime \prime}\left(z_{0}\right) z_{0}\right|^{-1 / 2}\left(r T^{-2}+r^{2+\varepsilon} T^{2 \xi-3}\right) \ll r T^{-2}+r^{2+\varepsilon} T^{2 \xi-3},
$$

and this bound also represents the true order of the contribution by standard arguments (see e.g., [4, Chapter 2] and [26, Lemma 4.6]). It follows that the integral in $(4.25)$, with $M(r ; T)$ replaced by $M^{*}(r ; T)$, converges for $\sigma>-1 / 2$, and the whole expression in (4.25) is

$$
\begin{aligned}
& \ll|1 / 2+i r-s|^{-1} r^{-1 / 2} \int_{T(r)}^{\infty}\left(r T^{-2}+r^{2+\varepsilon} T^{2 \xi-3}\right) T^{1 / 2-\sigma} d T \\
& \ll|1 / 2+i r-s|^{-1} r^{(\xi-2 \sigma) /(2-2 \xi)+\varepsilon} .
\end{aligned}
$$

If $r$ is not close to $t$, say $|r-t| \gg(t+1)^{\varepsilon}$ for $t \geq 0$, then this estimate can be improved on repeating the integration by parts over $T$ in (4.25). Indeed, the expression in (4.25) equals, for any fixed $\nu \in \mathbb{N}$,

$$
\begin{aligned}
& \frac{(-1)^{\nu} \Gamma^{2}(1 / 2-i r)}{\Gamma(1-2 i r)(1 / 2+i r-s) \ldots(1 / 2+\nu+i r-s)} \\
& \quad \times \int_{T(r)}^{\infty} T^{1 / 2+\nu+i r-s}\left(\frac{\partial}{\partial T}\right)^{\nu} M(r ; T) d T,
\end{aligned}
$$

plus integrated terms which are negligible. It can be checked by induction that the size of $(\partial / \partial T)^{\nu} M(r ; T)$ decreases by a factor of order $T^{-1}$ at each new integration by parts, while the oscillating factor remains the same. Hence analogously to the preceding discussion we obtain

$$
\left(\frac{\partial}{\partial T}\right)^{\nu} M(r ; T) \ll_{\varepsilon, \nu}\left(r T^{-2}+r^{2+\varepsilon} T^{2 \xi-3}\right) T^{-\nu},
$$


so that each integration by parts reduces the estimate of the expression in (4.25) by a factor $|r-t|^{-1}$. Therefore, repeating this process sufficiently many times, we may truncate the sum over $\kappa_{j}$ in (4.19) to $\left|\kappa_{j}-t\right| \ll(t+1)^{\varepsilon}$. The net result of this discussion is that if $\sigma \geq-1 / 2+\varepsilon, t \geq 0$ and $s=\sigma+i t$ is well separated from the poles of $\mathcal{Z}_{2}(s)$, then we have the bound

$$
\begin{aligned}
\int_{1}^{\infty} I_{2, d}\left(T, T^{\xi}\right) T^{-s} d T & \\
& \ll \sum_{\left|t-\kappa_{j}\right| \ll(t+1)^{\varepsilon}} \alpha_{j}\left|H_{j}^{3}(1 / 2)\right|(t+1)^{(\xi-2 \sigma) /(2-2 \xi)+\varepsilon}+1 .
\end{aligned}
$$

The right-hand side of (4.29) is clearly of polynomial order in $t$.

It should be remarked that the above argument can actually produce an asymptotic expansion for the left side of (4.29). Such a result may have applications in the context of the present paper. We shall return to this point elsewhere.

It remains to consider the term (see [24, p. 186])

$$
I_{2, c}\left(T, T^{\xi}\right)=\pi^{-1} \int_{-\infty}^{\infty} \frac{|\zeta(1 / 2+i r)|^{6}}{|\zeta(1+2 i r)|^{2}} \Lambda\left(r ; T, T^{\xi}\right) d r,
$$

which represents the contribution of the continuous spectrum to $\psi(T)$ in (4.15).

As an analogue of (4.19), we have

$$
\begin{array}{rl}
\int_{1}^{\infty} I_{2, c}\left(T, T^{\xi}\right) T^{-s} & d T \\
& =\pi^{-1} \int_{-\infty}^{\infty} \frac{|\zeta(1 / 2+i r)|^{6}}{|\zeta(1+2 i r)|^{2}} \int_{1}^{\infty} \Lambda\left(r ; T, T^{\xi}\right) T^{-s} d T d r
\end{array}
$$

provided that $\sigma$ is sufficiently large. This expression should be analytically continued to a meromorphic function in the half-plane $\sigma>-1 / 2$. Temporarily, we restrict $s$ to a horizontal strip of the form $|t|<1$ or $\tau<t<2 \tau$ for $\tau \gg 1$. Let us consider the latter case as an example.

First of all we note that if the range for $r$ in (4.30) is split up into the finite intervals $[\tau / 2,3 \tau]$ and the tails, then by previous arguments the contribution of the tails will be negligible because now $1 / 2+i r-s$ is well bounded away from zero.

In the remaining integral over $[\tau / 2,3 \tau]$, the integrand can be reduced to integrals of the type $X_{r}^{*}(s)$ in (4.24) except that the lower limit of the $T$-integration is changed to $T(\tau)$ in order to make it independent of $r$. Following now the previous argument, we integrate by parts over $T$ and simplify 
$M(r ; T)$ to $M^{*}(r ; T)$. Then we have the double integral

$$
\begin{aligned}
Y(s):= & \int_{\tau / 2}^{3 \tau}\left(\frac{\zeta^{3}(1 / 2+i r) \zeta^{3}(1 / 2-i r)}{\zeta(1+2 i r) \zeta(1-2 i r)}\right)\left(\frac{\Gamma^{2}(1 / 2-i r)}{\Gamma(1-2 i r)}\right) \\
& \times\left(1-\frac{i}{\sinh \pi r}\right)\left(\frac{1}{2}+i r-s\right)^{-1} \\
& \times \int_{T(\tau)}^{\infty} M^{*}(r ; T) T^{1 / 2+i r-s} d T d r
\end{aligned}
$$

to deal with, where $M^{*}(r ; T)$ is defined as in (4.27) except that the range for $z$ is now $\left[0, T \tau^{-a}\right]$. We rewrote the zeta-quotient in the integrand avoiding absolute values in order to make it analytic in $r$.

The function $Y(s)$ can be continued beyond the line $\sigma=1 / 2$, and analytic continuation to the strip $-1 / 2<\sigma<1 / 2$ is obtained by adding the term

$$
\begin{aligned}
-2 \pi\left(\frac{\zeta^{3}(s) \zeta^{3}(1-s)}{\zeta(2 s) \zeta(2-2 s)}\right)\left(\frac{\Gamma^{2}(s)}{\Gamma(2 s)}\right)( & \left.1-\frac{1}{\cos \pi s}\right) \\
& \times \int_{T(\tau)}^{\infty} M^{*}(i(1 / 2-s) ; T) d T
\end{aligned}
$$

to the integral (4.31). To see this we move the integration over $r$ in (4.31) to $\operatorname{Im} r=c(\tau)=(\log \tau)^{-3 / 4}$ plus two obvious vertical segments. In view of the zero-free region of $\zeta(s)$ (see [4, Chapter 6]) no singularities of the integrand (zeros of $\zeta(1 \pm 2 i r)$ ) will be encountered. The resulting expression $Y^{*}(s)$, say, yields an analytic continuation of $Y(s)$ to the domain

$$
\{s: \sigma>1 / 2\} \cup \mathcal{D},
$$

where

$$
\mathcal{D}=\{s: 1 / 2-c(\tau)<\sigma \leq 1 / 2, \tau \leq t \leq 2 \tau\} .
$$

Then to get a further continuation we suppose that $s$ is now inside $\mathcal{D}$. We move the integration over $r$ in $Y^{*}(s)$ back to the original interval on the real axis. In doing this we encounter only one singularity, namely the simple pole at $r=i(1 / 2-s)$, which by the residue theorem gives the term (4.32). The resulting integral, which has the same expression as (4.31) but with different $s$, is regular not only inside $\mathcal{D}$ but in the whole vertical strip $-1 / 2<\sigma<1 / 2$. We should note that when $s$ is on the line $\sigma=1 / 2$ our argument needs to be altered a little: we have to add an appropriate small indentation to the above segment on the real line. 
The function (4.32) has poles at the zeros of $\zeta(2 s)$, that is, at the points $\varrho / 2$ with $\varrho$ running over the complex zeros of Riemann's zeta-function, and these are also the only singularities of the function (4.30) for $\sigma>-1 / 2$. It remains to show that the latter function is of polynomial order for $\tau$ for $-1 / 2+\varepsilon \leq \sigma \leq 1-\varepsilon, \tau \leq t \leq 2 \tau$ if $\varepsilon$-neighbourhoods of the poles are excluded. For the function (4.31), the argument is as before (in place of summation over the discrete spectrum, we now have integration over a finite portion of the continuous spectrum).

As to the estimation of the function (4.32), the only problem that remains is the order of $1 / \zeta(s)$ in the critical strip when $s$ is separated from the zeros $\varrho$. However, it is easy to see that this is of polynomial order in $t$ by appealing to the well-known formula [26, Theorem 9.6(B)]

$$
\log \zeta(s)=\sum_{|t-\gamma| \leq 1} \log (s-\varrho)+O(\log t),
$$

where $\gamma=\operatorname{Im} \varrho$. This completes the proof of Theorem 3 .

REMARK. The preceding argument can be carried out even for the Mellin transform of the square of the Hecke $L$-function attached to a holomorphic cusp form, for an appropriate analogue of the decomposition (4.15) has been established by the third author [21]. The case of non-holomorphic cusp forms is more problematic, for instead of a precise analogue of (4.15), only an approximate variant due to the second author [14] is known. Nevertheless, the "barrier" $\sigma=1 / 2$ is breakable even in this case, for an analogue of (4.1) can be shown to hold for $\sigma>2 / 5$.

5. Further bounds for $\mathcal{Z}_{2}(s)$. One can obtain from Theorem 3 an explicit bound for $\mathcal{Z}_{2}(s)$, namely in terms of the value of the constant $B$ in (4.1). This constant depends on the contribution of the discrete and continuous spectrum. The former will yield a fairly explicit bound with a reasonable constant $B$ for $-1 / 2<\sigma<1 / 2$ (see (4.13) and (4.29)), while the latter will yield a poor bound for $B$. For $\sigma>1 / 2$ the situation is reversed. However, for $\sigma>1 / 2$ we can obtain better results by exploiting the integral representation (2.4). In this way we may obtain both pointwise and mean square bounds for $\mathcal{Z}_{2}(s)$ in the range $1 / 2<\sigma<1$. The results, which are gathered in Theorem 4 below, may be compared with those of Theorem 2 for $\mathcal{Z}_{1}(s)$. Naturally, they are weaker, since the function $\mathcal{Z}_{1}(s)$ is less difficult to deal with than $\mathcal{Z}_{2}(s)$.

For $\sigma=\operatorname{Re} s>1$ we have

$$
\mathcal{Z}_{2}(s)=\int_{1}^{X}|\zeta(1 / 2+i x)|^{4} x^{-s} d x+\int_{X}^{\infty}|\zeta(1 / 2+i x)|^{4} x^{-s} d x=I^{\prime}+I^{\prime \prime},
$$


say, where $X>1$ is a parameter to be suitably determined later. We have

$$
I^{\prime}=\int_{1}^{X}|\zeta(1 / 2+i x)|^{4} x^{-s} d x \ll X^{1-\sigma} \log ^{4} X \quad(1 / 2<\sigma<1),
$$

which follows by integration by parts and the classical bound for the fourth moment of $|\zeta(1 / 2+i x)|$. Next, for $s=\sigma+i t, \sigma>1, t \geq t_{0}>0, Q_{4}=P_{4}+P_{4}^{\prime}$,

$$
\begin{aligned}
I^{\prime \prime} & =\int_{X}^{\infty}|\zeta(1 / 2+i x)|^{4} x^{-s} d x=\int_{X}^{\infty}\left(Q_{4}(\log x)+E_{2}^{\prime}(x)\right) x^{-s} d x \\
& =X^{1-s} \sum_{j=1}^{5} \frac{Q_{4}^{(j-1)}(\log X)}{(s-1)^{j}}-E_{2}(X) X^{-s}+s \int_{X}^{\infty} E_{2}(x) x^{-s-1} d x,
\end{aligned}
$$

where we used the defining property (1.10) with $k=2$. Suppose now that $c$ is a constant for which

$$
E_{2}(T) \ll T^{c+\varepsilon}
$$

(currently we know (see (1.13)) that (5.3) holds with $c=2 / 3$ and that $c<1 / 2$ cannot work; it is conjectured that $c=1 / 2$ works). Since we have (cf. (1.13) or (2.19))

$$
\int_{0}^{T} E_{2}^{2}(t) d t \ll T^{2} \log ^{C} T \quad(C \leq 22),
$$

it follows that

$$
\begin{aligned}
\int_{T}^{2 T}\left|E_{2}(x)\right| x^{-\sigma-1} d x & \leq\left(\int_{T}^{2 T} E_{2}^{2}(x) d x \int_{T}^{2 T} x^{-2 \sigma-2} d x\right)^{1 / 2} \\
& \ll T^{1 / 2-\sigma} \log ^{11} T .
\end{aligned}
$$

Thus the last integral in (5.2) converges absolutely for $\sigma>1 / 2$, thereby providing analytic continuation of $\mathcal{Z}_{2}(s)$ in the half-plane $\sigma>1 / 2$. From (5.1)-(5.4) we then obtain

$$
\mathcal{Z}_{2}(s) \ll X^{1-\sigma} \log ^{4} X+t X^{1 / 2-\sigma} \log ^{11} X,
$$

and the choice $X=t^{2} \log ^{14} t$ ( $\sigma$ is assumed throughout to be fixed) gives

$$
\mathcal{Z}_{2}(s) \ll t^{2-2 \sigma}(\log t)^{18-14 \sigma} \quad(1 / 2<\sigma<1) .
$$

We now pass to mean square estimates, starting from

$$
I(s):=\int_{T}^{2 T}\left|\mathcal{Z}_{2}(\sigma+i t)\right|^{2} d t \leq \int_{T / 2}^{5 T / 2} \varphi(t)\left|\mathcal{Z}_{2}(\sigma+i t)\right|^{2} d t,
$$


where $1 / 2<\sigma<1$ as before, and $\varphi(t)$ is the test function appearing in Theorem 1. We have

$$
I(s) \ll I_{1}(s)+I_{2}(s),
$$

where using (5.2) we have

$$
\begin{aligned}
I_{1}(s):= & \left.\left.\int_{T / 2}^{5 T / 2} \varphi(t)\left|\int_{1}^{X}\right| \zeta(1 / 2+i x)\right|^{4} x^{-s} d x\right|^{2} d t, \\
I_{2}(s):= & \int_{T / 2}^{5 T / 2} \varphi(t) \mid X^{1-s} \sum_{j=1}^{5} \frac{Q_{4}^{(j-1)}(\log X)}{(s-1)^{j}} \\
& -E_{2}(X) X^{-s}+\left.s \int_{X}^{\infty} E_{2}(x) x^{-s-1} d x\right|^{2} d t .
\end{aligned}
$$

Note now that by $r$ integrations by parts we have

$$
\begin{aligned}
\int_{T / 2}^{5 T / 2} \varphi(t)\left(\frac{y}{x}\right)^{i t} d t & =(-1)^{r} \int_{T / 2}^{5 T / 2} \varphi^{(r)}(t) \frac{(y / x)^{i t}}{(i \log (y / x))^{r}} d t \\
& \ll_{r} T^{1-r}\left|\log \frac{y}{x}\right|^{-r} \ll T^{-A}
\end{aligned}
$$

for any fixed $A>0$ and any given $\varepsilon>0$, provided that $|y-x| \geq x T^{\varepsilon-1}$ and $r=r(A, \varepsilon)$ is large enough. By using (5.3) and (5.6) it follows that

$$
\begin{aligned}
I_{1}(s) & =\int_{1}^{X} \int_{1}^{X}|\zeta(1 / 2+i x) \zeta(1 / 2+i y)|^{4}(x y)^{-\sigma} \int_{T / 2}^{5 T / 2} \varphi(t)\left(\frac{y}{x}\right)^{i t} d t d x d y \\
& \ll 1+\int_{T / 2}^{5 T / 2} \int_{1}^{X}|\zeta(1 / 2+i x)|^{4} x^{-2 \sigma} \int_{x-x T^{\varepsilon-1}}^{x+x T^{\varepsilon-1}}|\zeta(1 / 2+i y)|^{4} d y d x d t \\
& \ll 1+T \int_{1}^{X}|\zeta(1 / 2+i x)|^{4} x^{-2 \sigma}\left(x T^{\varepsilon-1} \log ^{4} x+E_{2}\left(x+x T^{\varepsilon-1}\right)\right. \\
& \ll 1+T \int_{1}^{X}|\zeta(1 / 2+i x)|^{4} x^{-2 \sigma}\left(x T^{\varepsilon-1} \log ^{4} x+x^{c+\varepsilon}\right) d x \\
& \left.\ll T^{\varepsilon}\left(X^{2-2 \sigma}+T+T T^{\varepsilon-1}\right)\right) d x
\end{aligned}
$$

if $X \ll T^{B}(B>0)$, since $\int_{1}^{X}|\zeta(1 / 2+i x)|^{4} x^{-a} d x$ is bounded for $a>1$. 
To bound $I_{2}(s)$ we shall use again (5.6) to obtain

$$
\begin{aligned}
I_{2}(s) \ll & X^{2-2 \sigma}+T X^{2 c-2 \sigma+\varepsilon} \\
& +T^{2} \int_{T / 2}^{5 T / 2} \varphi(t) \int_{X}^{\infty}\left|E_{2}(x)\right| x^{-\sigma-1} \\
& \times \int_{x-x T^{\varepsilon-1}}^{x+x T^{\varepsilon-1}}\left|E_{2}(y)\right| y^{-\sigma-1} d y d x d t .
\end{aligned}
$$

We use the elementary inequality $|a b| \leq \frac{1}{2}\left(|a|^{2}+|b|^{2}\right)$ and the mean square bound (2.19) to infer that the inner integrals in the above bound are

$$
\begin{aligned}
& \ll \int_{X}^{\infty} x^{-2 \sigma-2} \int_{x-x T^{\varepsilon-1}}^{x+x T^{\varepsilon-1}}\left(E_{2}^{2}(x)+E_{2}^{2}(y)\right) d y d x \\
& \ll X^{1+\varepsilon-2 \sigma} T^{-1}+\int_{X / 2}^{\infty} y^{-2 \sigma-2} E_{2}^{2}(y) \int_{y /\left(1+T^{\varepsilon-1}\right)}^{y /\left(1-T^{\varepsilon-1}\right)} d x d y \\
& \ll X^{1+\varepsilon-2 \sigma} T^{-1} .
\end{aligned}
$$

Consequently

$$
I_{2}(s) \ll_{\varepsilon} X^{2-2 \sigma}+T X^{2 c-2 \sigma+\varepsilon}+T^{2+\varepsilon} X^{1-2 \sigma}
$$

and

$$
I(s) \ll_{\varepsilon} T^{\varepsilon}\left(X^{2-2 \sigma}+T+T X^{1+c-2 \sigma}+T^{2} X^{1-2 \sigma}\right) .
$$

Hence taking $X=T^{1 /(1-c)}$ it follows that

$$
I(s) \ll_{\varepsilon} T^{\varepsilon}\left(T+T^{(2-2 \sigma) /(1-c)}\right) \quad(1 / 2<\sigma<1),
$$

since $1 / 2<c<1$ yields

$$
T^{2} X^{1-2 \sigma}=T^{2} T^{1 /(1-c)} X^{-2 \sigma} \leq T \cdot T^{c /(1-c)} X^{-2 \sigma}=T X^{1+c-2 \sigma} .
$$

The above discussion shows that

$$
\int_{1}^{T}\left|\mathcal{Z}_{2}(\sigma+i t)\right|^{2} d t \ll_{\varepsilon} T^{\varepsilon}\left(T+T^{(2-2 \sigma) /(1-c)}\right) \quad(1 / 2<\sigma<1) .
$$

We shall now obtain another mean square bound for $\mathcal{Z}_{2}(s)$. We start from (5.1) and (5.2) to obtain

$$
\int_{T}^{2 T}\left|\mathcal{Z}_{2}(s)\right|^{2} d t \ll J_{1}(s)+T^{2} J_{2}(s)+X^{2-2 \sigma} \log ^{8} X+T E_{2}^{2}(X) X^{-2 \sigma},
$$


where

$$
\begin{aligned}
& J_{1}(s):=\left.\left.\int_{T}^{2 T}\left|\int_{1}^{X}\right| \zeta(1 / 2+i x)\right|^{4} x^{-s} d x\right|^{2} d t \\
& J_{2}(s):=\int_{T}^{2 T}\left|\int_{X}^{\infty} E_{2}(x) x^{-s-1} d x\right|^{2} d t .
\end{aligned}
$$

To bound $J_{1}(s)$ and $J_{2}(s)$ we use (1.1) similarly to (3.13) and the mean square bounds (1.5) (with $k=4)$ and (2.19). It follows that

$$
\begin{aligned}
& \int_{T}^{2 T}\left|\mathcal{Z}_{2}(s)\right|^{2} d t \\
& \quad \ll\left(X^{5 / 2-2 \sigma}+T^{2} X^{1-2 \sigma}+X^{2-2 \sigma} \log ^{8} X+T E_{2}^{2}(X) X^{-2 \sigma}\right) \log ^{C} X .
\end{aligned}
$$

Since $E_{2}(T) \ll T^{2 / 3} \log ^{C} T$, the choice $X=T^{4 / 3}$ gives then

$$
\int_{1}^{T}\left|\mathcal{Z}_{2}(\sigma+i t)\right|^{2} d t \ll T^{(10-8 \sigma) / 3} \log ^{C} T \quad(1 / 2 \leq \sigma \leq 1),
$$

while the eighth moment in the form given by (1.6) would improve this bound to

$$
\int_{1}^{T}\left|\mathcal{Z}_{2}(\sigma+i t)\right|^{2} d t \ll_{\varepsilon} T^{4-4 \sigma+\varepsilon} \quad(1 / 2 \leq \sigma \leq 1) .
$$

The preceding results are summarized in

Theorem 4. For $s=\sigma+i t, 1 / 2<\sigma<1, t \geq t_{0}$, we have

$$
\mathcal{Z}_{2}(s) \ll t^{2-2 \sigma}(\log t)^{18-14 \sigma} .
$$

If (5.3) holds then we have

$$
\int_{0}^{T}\left|\mathcal{Z}_{2}(\sigma+i t)\right|^{2} d t \ll_{\varepsilon} T^{\varepsilon}\left(T+T^{(2-2 \sigma) /(1-c)}\right) \quad(1 / 2<\sigma<1),
$$

while we also have unconditionally

$$
\int_{0}^{T}\left|\mathcal{Z}_{2}(\sigma+i t)\right|^{2} d t \ll T^{(10-8 \sigma) / 3} \log ^{C} T \quad(1 / 2<\sigma \leq 1, C>0) .
$$

As a final remark we shall show how to apply the mean square bound (5.8) to obtain a bound which complements (2.22). Namely if we assume that (2.21) holds, then we have

$$
\int_{0}^{T}|\zeta(1 / 2+i t)|^{8} d t \ll_{\varepsilon} T^{c+\omega+\varepsilon},
$$


where $c$ is the constant appearing in (5.3). Since (2.21) is known to hold unconditionally with $\omega=1$, then $(5.9)$ and $c=2 / 3$ yield the bound

$$
\int_{0}^{T}|\zeta(1 / 2+i t)|^{8} d t \ll{ }_{\varepsilon} T^{5 / 3+\varepsilon} .
$$

This is not a good result yet (the best known exponent for the bound in (5.10) is $3 / 2$ ), but there is hope that this approach may lead to improvements.

To prove (5.9) we use (2.7) with $s=1 / 2+i t$, moving the line of integration to $a=d$ with $1 / 2<d<1$ and $a=\varepsilon$ in portions containing $\mathcal{Z}_{2}(w)$ and $\sum_{j}$, respectively. We then obtain, using (2.9) and the Cauchy-Schwarz inequality,

$$
|\mathcal{G}(1 / 2+i t)|^{2} \ll T^{2 d+1+\varepsilon} t^{-2}\left(\int_{t-T^{\varepsilon}}^{t+T^{\varepsilon}}\left|\mathcal{Z}_{2}(d+i v)\right|^{2} d v+1\right)
$$

$$
(1 / 2<d<1) .
$$

Inserting (5.11) in the right-hand side of (2.21) we obtain

$$
\begin{aligned}
& \int_{T}^{2 T}|\zeta(1 / 2+i t)|^{8} d t \\
& \ll_{\varepsilon} T^{2 d-1+\varepsilon} \int_{T^{1 / 2}}^{T^{\omega+\varepsilon}}\left(\int_{t-T^{\varepsilon}}^{t+T^{\varepsilon}}\left|\mathcal{Z}_{2}(d+i v)\right|^{2} d v+1\right) d t+T^{1+\varepsilon} \\
& \quad \ll T^{2 d-1+\varepsilon} \int_{T^{1 / 2}-T^{\varepsilon}}^{T^{\omega+\varepsilon}+T^{\varepsilon}}\left|\mathcal{Z}_{2}(d+i v)\right|^{2} \int_{v-T^{\varepsilon}}^{v+T^{\varepsilon}} d t d v+T^{2 d+\omega-1+2 \varepsilon}+T^{1+\varepsilon} \\
& \ll T^{2 d-1+2 \varepsilon} \int_{T^{1 / 2}-T^{\varepsilon}}^{T^{\omega+\varepsilon}+T^{\varepsilon}}\left|\mathcal{Z}_{2}(d+i v)\right|^{2} d v+T^{2 d+\omega-1+2 \varepsilon}+T^{1+\varepsilon} .
\end{aligned}
$$

If we use the mean square bound (5.8) it follows that

$\int_{T}^{2 T}|\zeta(1 / 2+i t)|^{8} d t \ll_{\varepsilon} T^{2 d-1+\varepsilon}\left(T^{\omega}+T^{(2-2 d) \omega /(1-c)}\right)+T^{2 d+\omega-1+\varepsilon}+T^{1+\varepsilon}$.

Choose $T^{\omega}=T^{(2-2 d) \omega /(1-c)}$, that is, $d=\frac{1}{2}(c+1)$. Then $3 / 4 \leq d \leq 5 / 6$, and (5.9) follows.

\section{References}

[1] F. V. Atkinson, The mean value of the Riemann zeta-function, Acta Math. 81 (1949), 353-376. 
[2] H. Bateman and A. Erdélyi, Higher Transcendental Functions, Vol. 2, McGrawHill, New York, 1953.

[3] J. L. Hafner and A. Ivić, On the mean square of the Riemann zeta-function on the critical line, J. Number Theory 32 (1989), 151-191.

[4] A. Ivić, The Riemann Zeta-Function, Wiley, New York, 1985.

[5] -, Mean Values of the Riemann Zeta-Function, Tata Inst. Fund. Res. Lectures 82, Bombay, 1991 (distr. by Springer, Berlin).

[6] - The Mellin transform and the Riemann zeta-function, in: Proc. Conference on Elementary and Analytic Number Theory (Vienna, July 18-20, 1996), W. G. Nowak and J. Schoißengeier (eds.), Universität Wien \& Universität für Bodenkultur, Wien, 1996, 112-127.

[7] - On the ternary additive divisor problem and the sixth moment of the zetafunction, in: Sieve Methods, Exponential Sums, and their Applications in Number Theory, G. R. H. Greaves et al. (eds.), Cambridge Univ. Press, Cambridge, 1996, 205-243.

[8] - On the error term for the fourth moment of the Riemann zeta-function, J. London Math. Soc. (2) 60 (1999), 21-32.

[9] A. Ivić and Y. Motohashi, A note on the mean value of the zeta and L-functions VII, Proc. Japan Acad. Ser. A 66 (1990), 150-152.

[10] - - - The mean square of the error term for the fourth moment of the zetafunction, Proc. London Math. Soc. (3) 66 (1994), 309-329.

[11] - - - The fourth moment of the Riemann zeta-function, J. Number Theory 51 (1995), 16-45.

[12] M. Jutila, Mean value estimates for exponential sums, in: Number Theory (Ulm, 1987), Lecture Notes in Math. 1380, Springer, Berlin, 1989, 120-136.

[13] - , The fourth moment of Riemann's zeta-function and the additive divisor problem, in: Analytic Number Theory, Proc. of a Conference in Honor of H. Halberstam, Vol. 2, B. C. Berndt et al. (eds.), Birkhäuser, Boston, 1996, 517-536.

[14] -, Mean values of Dirichlet series via Laplace transforms, in: Proc. Taniguchi International Symposium on Analytic Number Theory, Kyoto, 1996, Y. Motohashi (ed.), Cambridge Univ. Press, Cambridge, 1997, 169-207.

[15] A. A. Karacuba and S. M. Voronin, The Riemann Zeta-Function, de Gruyter, Berlin, 1992.

[16] N. V. Kuznetsov, Sums of Kloosterman sums and the eighth moment of the Riemann zeta-function, in: Papers presented at the Ramanujan Colloquium, Bombay, 1989, publ. for Tata Institute (Bombay) by Oxford Univ. Press, Oxford, 1989, 57117.

[17] N. N. Lebedev, Special Functions and their Applications, Dover, New York, 1972.

[18] Y. Motohashi, Spectral mean values of Maass waveform L-functions, J. Number Theory 42 (1992), 258-284.

[19] - , The fourth power mean of the Riemann zeta-function, in: Proc. of the Amalfi Conference on Analytic Number Theory, 1989, E. Bombieri et al. (eds.), Università di Salerno, Salerno, 1992, 325-344.

[20] - An explicit formula for the fourth power mean of the Riemann zeta-function, Acta Math. 170 (1993), 181-220.

[21] - The mean square of Hecke L-series attached to holomorphic cusp forms, RIMS Kyoto Univ. Kokyuroku 886 (1994), 214-227.

[22] - , A relation between the Riemann zeta-function and the hyperbolic Laplacian, Ann. Scuola Norm. Sup. Pisa Cl. Sci. (4) 22 (1995), 299-313. 
[23] Y. Motohashi, The Riemann zeta-function and the non-Euclidean Laplacian, Sugaku Expositions 8 (1995), 59-87.

[24] —, Spectral Theory of the Riemann Zeta-Function, Cambridge Univ. Press, Cambridge, 1997.

[25] E. C. Titchmarsh, Introduction to the Theory of Fourier Integrals, Clarendon Press, Oxford, 1948

[26] —, The Theory of the Riemann Zeta-Function, Clarendon Press, Oxford, 1951.

Katedra Matematike RGF-a

Universiteta u Beogradu

Djušina 7, 11000 Beograd

Serbia (Yugoslavia)

E-mail: aleks@ivic.matf.bg.ac.yu aivic@rgf.rgf.bg.ac.yu

Department of Mathematics

University of Turku

FIN-20014 Turku, Finland

E-mail: matti.jutila@utu.fi
Department of Mathematics College of Science and Technology Nihon University, Surugadai Tokyo-101, Japan E-mail: ymoto@math.cst.nihon-u.ac.jp

Received on 29.9.1998

and in revised form 31.1.2000 